J. Inst. Math. Jussieu (2021), 1-29

doi:10.1017/S1474748021000517 C The Author(s), 2021. Published by Cambridge University Press. 1 This is an Open Access article, distributed under the terms of the Creative Commons Attribution licence (https://creativecommons.org/licenses/by/4.0), which permits unrestricted re-use, distribution and reproduction, provided the original article is properly cited.

\title{
AN APERIODIC TILE WITH EDGE-TO-EDGE ORIENTATIONAL MATCHING RULES
}

\author{
JAMES J. WALTON® ${ }^{1}$ AND MICHAEL F. WHITTAKER (iD ${ }^{2}$ \\ ${ }^{1}$ School of Mathematical Sciences, University of Nottingham, University Park, \\ Nottingham NG7 2RD, United Kingdom \\ (james.walton@nottingham.ac.uk) \\ ${ }^{2}$ School of Mathematics and Statistics, University of Glasgow, University Place, \\ Glasgow Q12 8QQ, United Kingdom \\ (Mike.Whittaker@glasgow.ac.uk)
}

(Received 17 December 2020; revised 20 September 2021; accepted 22 September 2021)

\begin{abstract}
We present a single, connected tile which can tile the plane but only nonperiodically. The tile is hexagonal with edge markings, which impose simple rules as to how adjacent tiles are allowed to meet across edges. The first of these rules is a standard matching rule, that certain decorations match across edges. The second condition is a new type of matching rule, which allows tiles to meet only when certain decorations in a particular orientation are given the opposite charge. This forces the tiles to form a hierarchy of triangles, following a central idea of the Socolar-Taylor tilings. However, the new edge-to-edge orientational matching rule forces this structure in a very different way, which allows for a surprisingly simple proof of aperiodicity. We show that the hull of all tilings satisfying our rules is uniquely ergodic and that almost all tilings in the hull belong to a minimal core of tilings generated by substitution. Identifying tilings which are charge-flips of each other, these tilings are shown to have pure point dynamical spectrum and a regular model set structure.
\end{abstract}

Key words and phrases: aperiodic tilings, fractal, monotile, nonperiodic

2020 Mathematics subject classification: Primary 52C23

Secondary 37E25; 05B45

\section{Introduction}

The fact that periodically arranged structures can be enforced by local rules is familiar to everyone. In covering the plane with unit squares so that squares must meet edge-toedge, a periodic tessellation results. This simple principle of local constraints enforcing global structure explains how crystalline structures can form. Therefore, it was a great surprise to crystallographers in the 1980s when Dan Shechtman discovered a metal alloy whose diffraction pattern implied a great deal of structural order but had rotational symmetry precluding periodicity [20]. Since the atomic organization must 
still result from local interactions, the question arises of how such aperiodic patterns can result from only local rules. In the other direction, it is known that hierarchical aperiodic patterns generated by a substitution rule can be forced from local matching rules $[7,8,14]$.

Already in the 1960s, it was observed by Robert Berger in solving Hao Wang's Domino Problem [24] that one may find square tiles with decorated edges that can tile the plane but only nonperiodically [5, 18]. The first such set that he found had 20,426 tiles, initiating the hunt to find smaller aperiodic tile sets [9]. The most famous, and arguably the most beautiful, such tile set is the pair of tiles discovered by Roger Penrose in the 1970s [15]. Isometric copies of these edge-decorated tiles (represented either as a pair of thick and thin rhombs or as kite and dart tiles) can tile the plane but only aperiodically, and in fact form highly structured repetitive tilings with striking 10-fold rotational structure, similar to the rotational symmetry of the diffraction patterns of quasicrystals first observed by Shechtman.

Naturally, one wonders if two tiles are needed. The 'monotile problem' asks: Is there a single tile of the Euclidean plane for which copies of the tile can be used to tile the plane, but only nonperiodically? There are several ways to interpret this question. By 'copies' of the tile, one usually means isometric copies of the tile, through rotations, reflections, and translations, although it is also of great interest to allow only rotations and translations [9]. There are several demands one could make of such a tile. It is natural, for example, to ask that the tile not have too wild a shape: it should be the closure of its interior, but one might also demand that it is a polytope, just a topological disc, or perhaps merely that it has connected interior. And finally, by 'tiling the plane' one usually means that the tiles cover the plane but that distinct tiles overlap on at most their boundaries (however, we note here Gummelt's aperiodic tile that tiles the plane with overlaps [10]). One should also specify what rules are permitted in how tiles can be placed next to each other should these rules be forced by geometry alone, are color matchings permitted, or can more complicated local rules be specified?

The best current solution to the monotile problem without overlapping tiles is the Socolar-Taylor tile [21, 22, 23]. This tile forces limit-periodic structures, closely related to (but distinct from) the $\left(1+\epsilon+\epsilon^{2}\right)$-tilings $[16,17]$ and half-hex tilings [1], using just a single tile. However, the matching rules restrict configurations of not only neighboring tiles but also nontouching next-nearest neighbors. An alternative form of the tile may be given which has nearest-neighbor locality, but at the cost that the tile has a complicated shape, with disconnected interior. So the hunt remains for a monotile with simple shape but also next-nearest-neighbor matching rules.

In this paper we define a new aperiodic tile which, like the first form of the SocolarTaylor tile, satisfies the requirement that it is a simple geometric shape, again being a hexagon. Unlike the Socolar-Taylor tile, the rules for which tiles are allowed to meet are nearest-neighbor, and in fact only need to be checked on pairs of tiles meeting along an edge. The drawback is that although these rules are simply stated and entirely local (in fact, edge-to-edge), they cannot be enforced by shape alone. Rather, whether two tiles can meet is determined by orientation as well as 'charge' (equivalently, decoration of one 


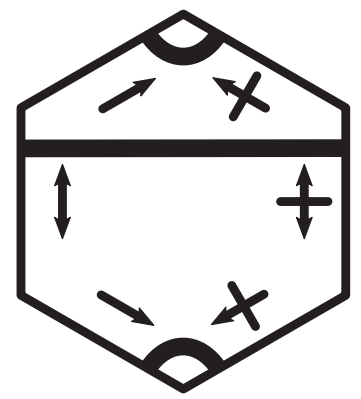

Figure 1. The tile. It consists of one horizontal straight R1-segment and two R1-turning segments, meeting the edges with the same offsets from the center axes. The left-hand edges are labeled with negative R2-charges, which are oriented, respectively from top to bottom, clockwise, both clockwise and counterclockwise, and counterclockwise. The right-hand edges are labeled with positive R2-charges, oriented, from top to bottom, counterclockwise, both clockwise and counterclockwise, and clockwise.

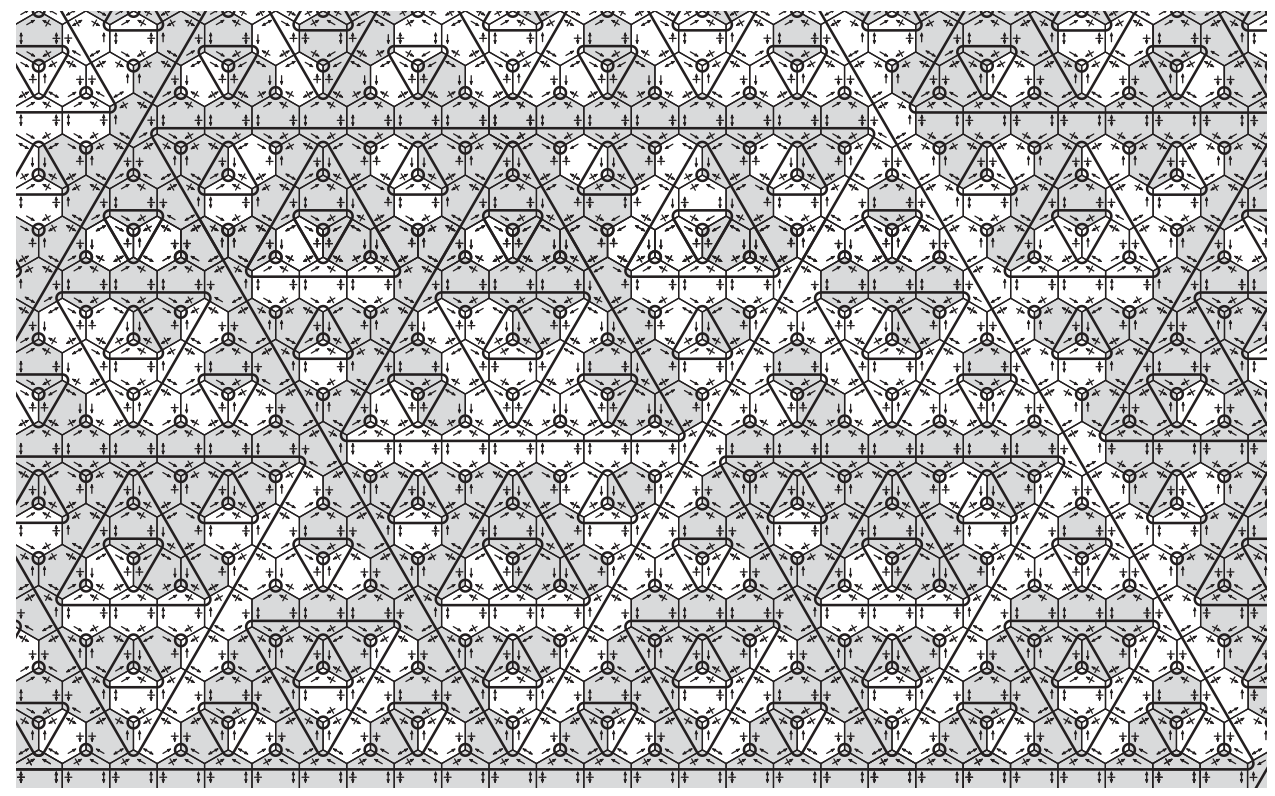

Figure 2. Patch of a valid tiling, where reflections of the tile of Figure 1 are shaded in gray.

of two colors) along edges. Our tile is given in Figure 1 . Two tiles $t_{1}$ and $t_{2}$ are permitted to meet along a shared edge $e$ only if

$\mathbf{R} 1$ the decorations of black lines of $t_{1}$ and $t_{2}$ are continuous across $e$ and

$\mathbf{R 2}$ whenever the two charges at $e$ in $t_{1}$ and $t_{2}$ both have a clockwise orientation, they are opposite in charge. 
Throughout, we shall call a tiling of the plane by isometric copies of the single tile of Figure 1 valid when tiles meet edge-to-edge and satisfy rules $\mathbf{R} \mathbf{1}$ and $\mathbf{R 2}$ at each edge, see Figure 2. Our main theorem is that such tilings exist, and that they are always nonperiodic:

Theorem 1.1. There exist valid tilings by the tile of Figure 1. Moreover, any valid tiling $T$ is nonperiodic - that is, if $T=T+x$ for $x \in \mathbb{R}^{2}$, then $x=0$.

As we shall show in $\S 4$, the $\mathbf{R} 1$ rule alone forces $\mathbf{R} 1$-triangles to contain a particular hierarchical nesting of others, just as in the Socolar-Taylor tilings. The way that arbitrarily large such triangles are forced with the new rule $\mathbf{R 2}$, however, is quite different. Indeed, first, the matching rules being edge-to-edge allows for tilings with 'infinite fault lines', as well as some other defects, as discussed in $§ 4$. Second, the patterns of tile parities (given by labeling hexagons only with the information of whether the tile of Figure 1 or its mirror image is used) are very different, and in fact closely follow the structure of the R1-edges, which are forced to carry the same parities across them.

This observation leads to a remarkably simple proof of aperiodicity, presented in full detail in $\S 2$ and which we briefly outline now. It is easily seen that following one R1edge forward to a second one, belonging to an exterior triangle, flips the charges of the R1-edges (Lemma 2.1). This implies that the second R1-edge is longer (Lemma 2.2); the alternative would lead to a spiral of edges ending in a period 3 cycle (Figure 4), resulting in a parity mismatch. Hence there is no upper limit on the lengths of the edges of R1-triangles (Corollary 2.3), from which nonperiodicity quickly follows.

Although the central objective of the paper is to showcase a tile admitting a novel and elementary proof of aperiodicity, in the second half of the paper we proceed to further analyze the collection of valid tilings and its dynamics. Analogously to the Socolar-Taylor tilings, there are a particular pair of 'defect' tilings. The underlying R1-decorations can be completed with charges to make a valid tiling in eight different ways, of which the two with 3 -fold rotational symmetry are the nonrepetitive defects, each containing one instance of a particular vertex configuration. For the Socolar-Taylor tile, these defects are forced by the existence of such a vertex configuration, which does not appear in any other valid tiling. For our tile, this vertex in fact forces either this defect tiling or, alternatively, what we call an ' $n$-cycle'. There also exist tilings with 'fault lines', whose existence is linked to the fact that our matching rules are entirely edge-to-edge: two half-planes of valid tilings either side of a bi-infinite straight R1-edge may, up to a charge-flip of one side, be pasted together along the $\mathbf{R} 1$-edge. We give a classification result listing the possible R1-decorations of valid tilings in Theorem 4.5, which, like the proof of aperiodicity, is proven very directly from the simple structures of the $\mathbf{R} \mathbf{1}$-edges, in particular the $\mathbf{R} \mathbf{1}$-edge graph, introduced in $§ 3.2$. There is a simple method of adding $\mathbf{R 2}$ charge decorations to the $\mathbf{R} 1$-edge graph to make a valid tiling.

In $\$ 5$ we introduce a substitution rule which generates valid tilings. Using our classification result, one sees that defects can only ever appear sparsely and that all valid tilings have some form of supertile decomposition (Proposition 5.4). This allows us to deduce the existence of uniform patch frequencies, or equivalently, unique ergodicity of the associated tiling dynamical system. With respect to the unique invariant measure, 
almost all tilings belong to the minimal core of substitution tilings. This minimal hull is a 2-fold cover of another hull of tilings, also generated by substitution, given by identifying tilings which are charge-flips of each other. We are able to identify the multiplicity of the fibers of the map to the maximal equicontinuous factor, which demonstrates that the system is quite distinct from the Socolar-Taylor and Penrose $\left(1+\epsilon+\epsilon^{2}\right)$-tilings. The substitutional hull (modulo charge-flip) factors almost everywhere 1-to-1 to its maximal equicontinuous factor and so has pure point dynamical spectrum and the structure of a regular model set.

\section{Aperiodicity}

In this section we show that all valid tilings by the tile of Figure 1 are nonperiodic. We begin by defining the R1-triangles and how one associates charges to their edges.

\subsection{R1-triangles}

The straight R1-segments of the tiles are offset from the center axis of the tile, so we may assign them a direction. We choose for them to point to the right when the R1-segment is horizontal and offset toward the top of the tile - that is, when positioned as in Figure 1. The turns in the R1-lines (the small sections of decorations about two corners of the tile) are correspondingly offset, which means that they always turn leftward from the direction of a straight R1-segment leading into them. A maximal straight section of an R1-line will be called an $\boldsymbol{R} \mathbf{1}$-edge. Since turns are always to the left, the $\mathbf{R} \mathbf{1}$-edges always form either infinite lines (possibly composed of two edges, broken by a single turn) or triangles of three edges of the same length. With our convention of directing edges, triangles are always directed counterclockwise.

One may show more, namely that triangle edges must consist of $2^{n}-1$ straight sections, where $n \in \mathbb{N}$, and that there is a hierarchical and identical formation of $\mathbf{R} 1$-triangles inside every R1-triangle of the same size. These observations will not be necessary for our proof, although they will be proved in $\S 4$ when we investigate the set of all possible valid tilings.

\subsection{Charges of triangle edges}

The region to the immediate left of a directed $\mathbf{R} \mathbf{1}$-edge (even if it is infinite) is considered the 'inside' of the corresponding (possibly infinite) triangle. On a tile carrying an R1-edge, precisely one clockwise-oriented charge lies on the inside of the triangle, either positive or negative. We assign this charge also to the straight R1-segment. So, for example, translates and rotates of the tile of Figure 1 carry a negative charge, and its reflection carries a positive charge. It is easy to see that two consecutive straight $\mathbf{R} 1$-segments must be assigned the same charge, so we may consistently assign a charge $\operatorname{ch}(E) \in\{+,-\}$ to an entire R1-edge $E$. Given a charge $c$, we let $c^{*}$ be its opposite - that is, $+^{*}=-$ and $-{ }^{*}=+$.

Take an R1-triangle edge $E_{1}$ that, following its orientation forward, ends at a turn. The tile containing the turn carries a different R1-edge $E_{2}$. In this case we say that $E_{1}$ leads to $E_{2}$ and write $E_{1} \dashv E_{2}$. We further specify that $E_{1} \dashv^{N} E_{2}$ if $E_{2}$ is offset near to $E_{1}$, and that $E_{1} \dashv^{F} E_{2}$ if $E_{2}$ is offset far from $E_{1}$. Equivalently, we have that $E_{1} \dashv^{N} E_{2}$ 

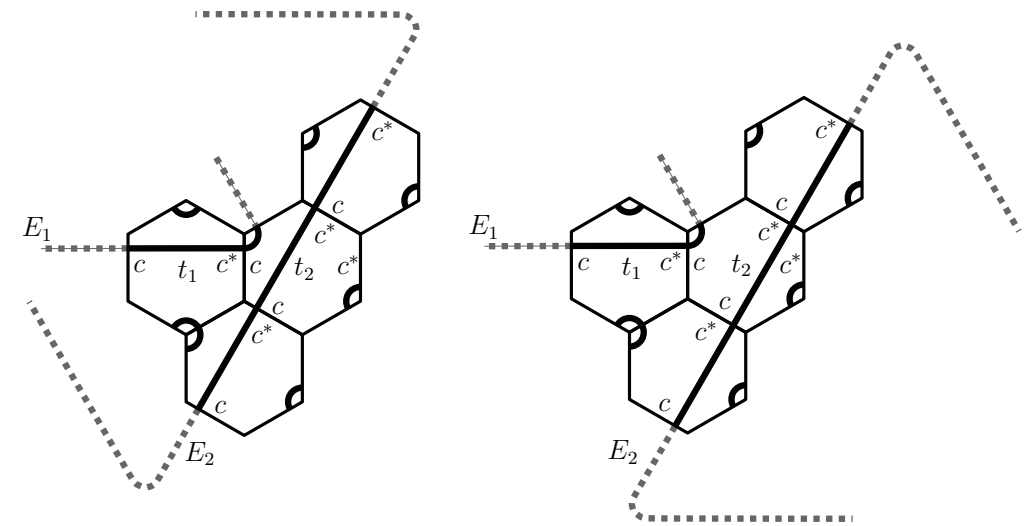

Figure 3. The definition of $E_{1} \dashv^{N} E_{2}$ (left) and $E_{1} \dashv^{F} E_{2}$ (right). Relevant charges and tiles $t_{1}$ and $t_{2}$ are indicated as used in the proof of Lemma 2.1.

(resp., $E_{1} \dashv^{F} E_{2}$ ) if the triangle with edge $E_{1}$ is contained in (resp., is not contained in) the triangle with edge $E_{2}$ (see Figure 3).

Lemma 2.1. In any valid tiling, if $E_{1} \dashv^{N} E_{2}$, then $\operatorname{ch}\left(E_{1}\right)=\operatorname{ch}\left(E_{2}\right)$, and if $E_{1} \dashv^{F} E_{2}$, then $\operatorname{ch}\left(E_{1}\right)=\operatorname{ch}\left(E_{2}\right)^{*}$. In particular, there is no chain $E_{1} \dashv^{F} E_{2} \dashv^{F} E_{3} \dashv^{F} E_{1}$ of three edges.

Proof. The proof follows from a simple inspection of Figure 3. Indeed, suppose that $E_{1} \dashv^{N} E_{2}$. Let $t_{1}$ be the tile containing the final straight R1-segment of $E_{1}$ before the turn and $t_{2}$ the tile containing the turn as well as a straight section of $E_{2}$. Let $e$ be the edge shared by the tiles $t_{1}$ and $t_{2}$. The charge on $e$ in $t_{1}$ is also clockwise-oriented and equal to $c^{*}$, and the charge on $e$ in $t_{2}$ is clockwise-oriented and thus equal to $\left(c^{*}\right)^{*}=c$. By definition, this charge is equal to $\operatorname{ch}\left(E_{2}\right)$, as required. The case for $E_{1} \dashv^{F} E_{2}$ is analogous; in this case, $\operatorname{ch}\left(E_{2}\right)$ is given by the charge of the edge opposite $e$ in $t_{2}$, which is $c^{*}$.

Given $E_{1} \dashv^{F} E_{2} \dashv^{F} E_{3} \dashv^{F} E_{1}$, by the foregoing we have $\operatorname{ch}\left(E_{1}\right)=\operatorname{ch}\left(E_{1}\right)^{* * *}=\operatorname{ch}\left(E_{1}\right)^{*}$ - a contradiction - so there is no such chain of three edges in a valid tiling.

\subsection{Finding edges of increasing length}

We let $L(E) \in \mathbb{N} \cup\{\infty\}$ be the length of an R1-edge $E$, the number of tiles containing the straight segments of $E$ (so not including the turning tiles).

Lemma 2.2. Consider $\boldsymbol{R} 1$-edges $E_{1} \dashv E_{2}$ in a valid tiling. Let $t$ denote the tile containing the terminating turn of $E_{1}$ and thus also a tile of $E_{2}$. Consider the collection $R$ of all tiles containing straight $\boldsymbol{R} 1$-segments of $E_{2}$ starting from and including $t$ and heading to the right from $E_{1}$. Then $\# R=\infty$ if $L\left(E_{1}\right)=\infty$, and $\# R>L\left(E_{1}\right)$ otherwise.

Proof. Suppose, to the contrary, that $\# R<L\left(E_{1}\right)=\infty$ or $\# R \leq L\left(E_{1}\right)<\infty$. We define a sequence $E_{1}, E_{2}, E_{3}, \ldots$ of edges of respective triangles $\Delta_{1}, \Delta_{2}, \Delta_{3}, \ldots$, which we will show must spiral inward and eventually form a 3-periodic chain, contradicting the previous 


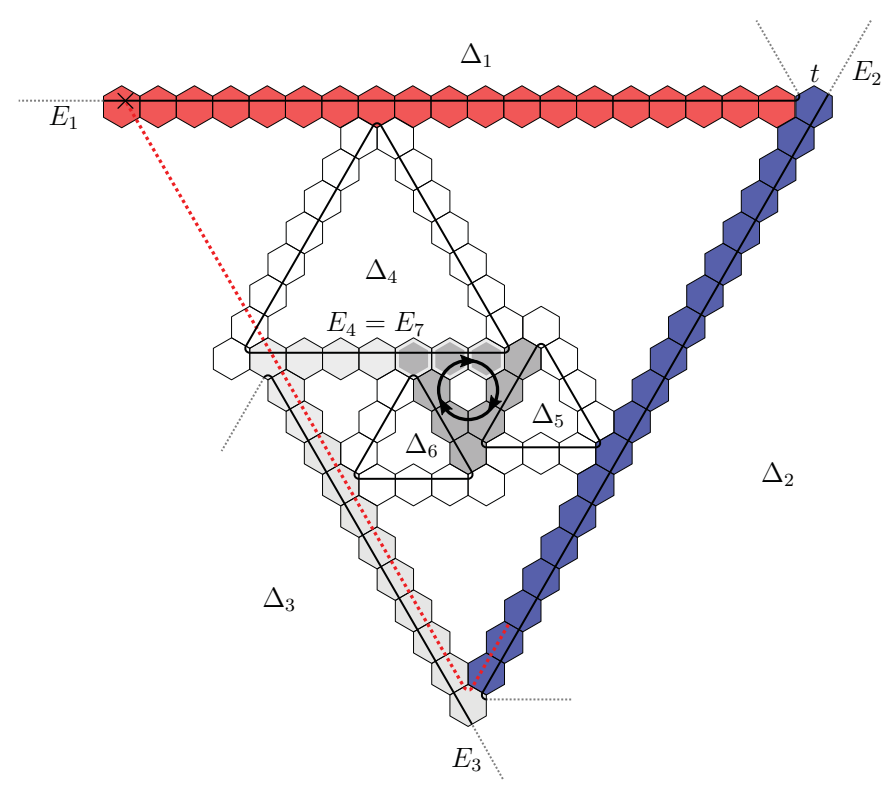

Figure 4. Creating a spiral of edges from an edge $E_{1}$ (red tiles) containing tiles greater than or equal to the number of tiles of $R$ (blue tiles) in $E_{2}$, as in the proof of Lemma 2.2.

lemma (see Figure 4). The edges $E_{1}$ and $E_{2}$ are already as given, and having constructed $E_{i}$, we define $E_{i+1}$ by following $E_{i}$ rightward from $E_{i-1}$ to its terminating turn, which is the tile containing $E_{i+1}$. Let $R_{i}$ be the collection of tiles containing straight R1-segments of each $E_{i}$, starting from the tile with the terminating turn of $E_{i-1}$ up to the terminating turn of $E_{i}$.

We observe that $E_{1} \dashv^{F} E_{2}$. Indeed, otherwise, the edge $E_{3}$ following $E_{2}$ would be part of the same R1-triangle; in particular, it would have length the same as that of $E_{2}$, causing it to intersect $E_{1}$ (see the red dotted line in Figure 4). Hence $E_{2}$ has orientation making $E_{2} \dashv E_{3}$. To prevent $E_{3}$ from intersecting $E_{1}$, it is necessary that $\# R_{3} \leq \# R_{2} \leq L\left(E_{2}\right)$. So $E_{2} \dashv E_{3}$ and $\# R_{3} \leq L\left(E_{2}\right)<\infty$. These properties of $E_{2}$ and $E_{3}$ are analogous to our initial assumptions on $E_{1}$ and $E_{2}$, so the argument repeats, showing that $E_{i} \dashv^{F} E_{i+1}$ for all $i \in \mathbb{N}$ and that $\# R_{i}$ is monotonically decreasing in $i$.

For $i \in \mathbb{N}$, the edge $E_{i+3}$ is parallel to $E_{i}$. All triangles $\Delta_{j}$ are in the exteriors of each other by the foregoing, so we see that $\# R_{i}=\# R_{i+3}$ is possible only if $E_{i}=E_{i+3}$. Indeed, $R_{i+3}$ must belong to the triangular region bounded between $\Delta_{i}, \Delta_{i+1}$, and $\Delta_{i+2}$, which has strictly fewer than $\# R_{i}$ tiles in each row parallel to $E_{i}$, except for the row containing $E_{i}$ itself. Since $\# R_{i}$ cannot strictly decrease indefinitely, we must have $E_{i}=E_{i+3}$ for sufficiently large $i$. But this contradicts Lemma 2.1, since we have found a chain $E_{i} \dashv^{F}$ $E_{i+1} \dashv^{F} E_{i+2} \dashv^{F} E_{i}$.

Corollary 2.3. In any valid tiling, there is no finite upper bound on the length of $\boldsymbol{R} \mathbf{1}$ edges. 
Proof. Supposing otherwise, we may find a finite triangle of largest size, say with edge $E$. Then $E \dashv E^{\prime}$ for some edge $E^{\prime}$, but Lemma 2.2 implies that $L(E)<L\left(E^{\prime}\right)$, a contradiction. So either there is an infinite R1-line or all triangles are finite but of unbounded size, as required.

Theorem 2.4. Any valid tiling $T$ is nonperiodic.

Proof. By Corollary 2.3, a valid tiling $T$ contains either an infinite R1-line or triangles of arbitrarily large size. In the latter case, $T$ is nonperiodic, since any given translation will not be able to transfer sufficiently large triangles to others.

So we just need to show that any tiling $T$ with an infinite R1-line is nonperiodic. Assume that $T$ contains an infinite line $L$ with no turn. Orient the tiling so that $L$ points to the right and consider the set $A=\left\{\Delta_{i}: i \in \mathbb{Z}\right\}$ of triangles $\Delta_{i}$ that share turning tiles with $L$ (see Figure 5). Suppose that there is some $\Delta \in A$ of largest size. Supposing it is finite, follow its edge $E$ which heads upward and to the right from $L$, leading to an edge $E^{\prime}$ belonging to triangle $\Delta^{\prime}$. The section of tiles from $t$ along $E^{\prime}$ toward $L$ has more tiles than $L(E)$ by Lemma 2.2, so in fact $\Delta^{\prime}$ must reach a tile of $L$ and hence $\Delta^{\prime} \in A$ too. But evidently $\Delta^{\prime}$ is larger than $\Delta$, contradicting our assumption that $\Delta$ is the largest. So either $\Delta$ is infinite in size or there is no largest size of triangle in $A$. In either case, $T$ cannot be periodic.

Suppose instead that $L$ has a turn. Let $E_{1}$ be the infinite-length edge of $L$ directed toward the turn and $E_{2}$ be the edge after the turn. We have $E_{1} \dashv E_{3}$ for another edge $E_{3}$, which is also infinite in length by Lemma 2.2. We thus have three infinite R1-edges,

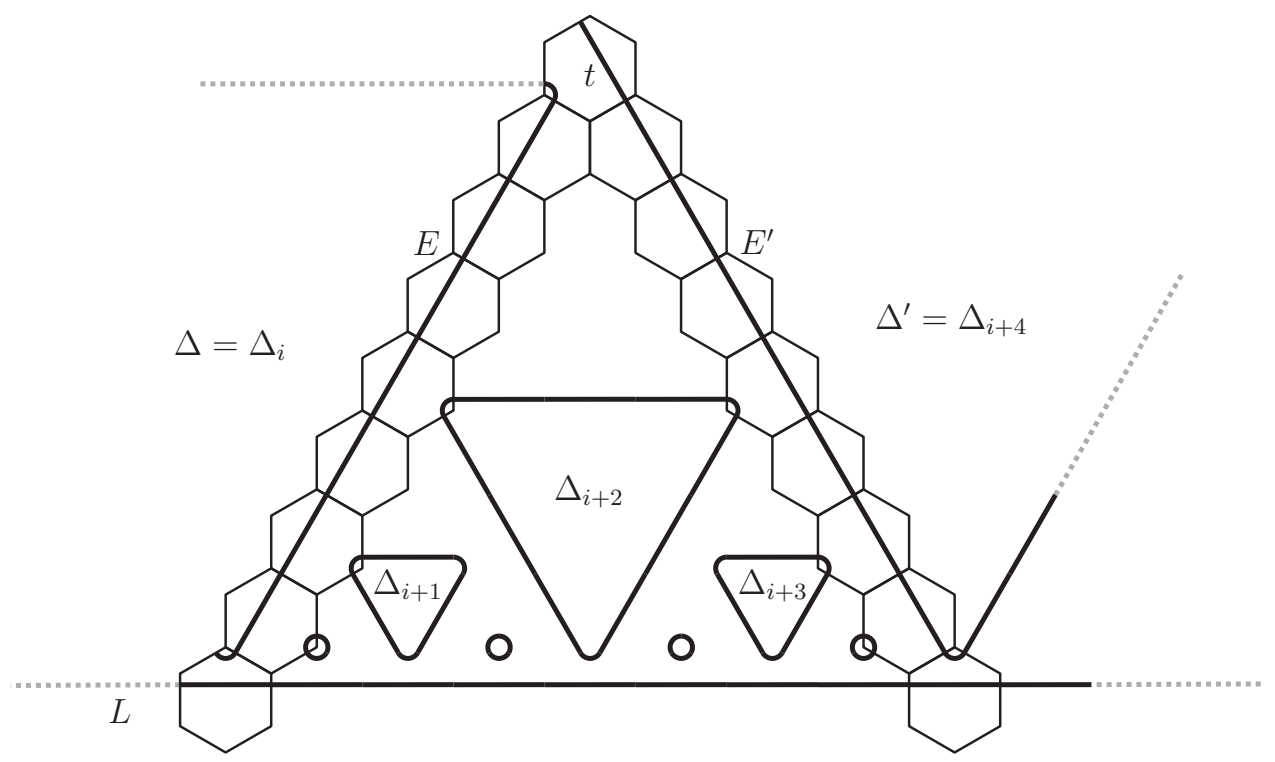

Figure 5. Proof of aperiodicity in case of the existence of an infinite R1-line $L$. 
and it is easy to see that any nontrivial translation would cause one to intersect another, nonparallel one, so $T$ is nonperiodic.

\section{Existence}

\subsection{Standard R1-tilings}

We now prove that tilings satisfying $\mathbf{R} 1$ and $\mathbf{R} 2$ exist. We begin by giving a class of tilings with 'standard' R1 decorations of hierarchically positioned R1-triangles, which we later show can also be equipped with $\mathbf{R 2}$ decorations making valid tilings.

Definition 3.1. The size $s(\Delta)$ of an R1-triangle is defined to be $L(E)+1$, where $E$ is an R1-edge of $\Delta$. A loop of three R1-turns is also considered to be an R1-triangle, with size 1 .

Definition 3.2. For each $n \in \mathbb{N}_{0}$, we define a standard patch $P_{n}$ of hexagonal tiles with (partial) R1-decorations. We begin with an R1-triangle $\Delta$ of size $2^{n}$ (see the left-hand patch of Figure 6). Another triangle $\Delta^{\prime}$ of size $2^{n-1}$ is placed inside of $\Delta$ in the only way possible - that is, with edges leading to the centers of edges of $\Delta$ (see the second patch of Figure 6). This leaves four triangular regions bounded between the edges of $\Delta$ and $\Delta^{\prime}$. We repeat the procedure by placing four triangles of size $2^{n-2}$, one in each region with edges meeting the edges bounding the region (see the third patch of Figure 6). We continue until triangles of size 1 are placed (see the fourth patch of Figure 6). The tiles carrying the R1-triangle $\Delta$ and its interior, with R1-decorations as constructed here, define the patch $P_{n}$.

Remark 3.3. The previous definition uniquely defines $P_{n}$, up to translation and rotation. Notice that all interior tiles of the patch $P_{n}$ are given full R1-decorations, but the tiles meeting the boundary are only partially decorated.

Although not needed for the arguments of this section, this hierarchical pattern of triangles is forced by the rule $\mathbf{R} \mathbf{1}$, as we shall see in Proposition 4.1. We now consider a natural collection of tilings associated to these standard patches:

Definition 3.4. An $\boldsymbol{R} 1$-tiling is a tiling of hexagons decorated with R1-decorations (but not $\mathbf{R 2}$-decorations) which satisfies $\mathbf{R} 1$. Such a tiling is called a standard $\boldsymbol{R} \mathbf{1}$-tiling

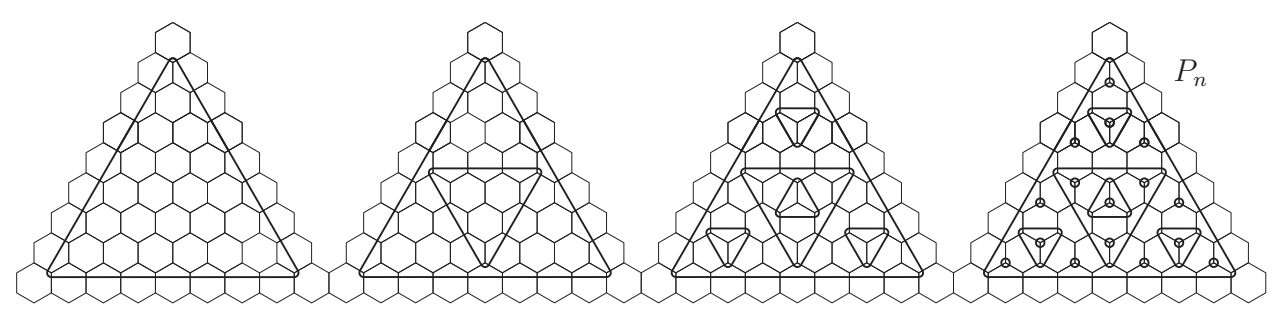

Figure 6. Construction of a standard patch $P_{n}$, here $P_{3}$. Starting with a triangle of size $2^{n}$, triangles of size $2^{i}$ are added for decreasing $i$ until ones of size 1 are placed, defining $P_{n}$. 
if every finite patch is contained in a translate or rotate of a standard patch $P_{n}$. The collection of all standard R1-tilings is denoted by $\Omega_{\mathrm{a}}$.

In the foregoing definition, one could allow $P_{n}$ to be extended to full R1-decorations on boundary tiles (so that the given subpatch may share boundary tiles). However, this does not affect which R1-tilings are standard. Indeed, a copy of $P_{n}$ is embedded in $P_{m}$ for any $m \geq n$ and in the interior of $P_{m}$ if $m \geq n+2$. Such embeddings also make it clear that standard R1-tilings exist, by choosing a nested union of such patches covering the entire plane.

\section{Remark 3.5.}

(1) We denote the collection of standard R1-tilings by $\Omega_{\mathrm{a}}$ because these tilings are easily seen to be mutually local derivable (MLD; see [2]) to the arrowed hex tilings [1]. Alternatively, these tilings are precisely those whose R1-decorations come from the Socolar-Taylor tilings [21].

(2) These tilings can be constructed by substitution rules, either from the arrowed halfhex substitution via pseudo-inflations (see [1]) or from an associated stone inflation on triangle tilings as described in $\S 5$.

\subsection{R1-edge graphs}

Here we shall see that all $\mathbf{R} 1$-tilings satisfying certain restrictions on the structure of the R1-edges (which includes any tiling in $\Omega_{\mathrm{a}}$ ) may always be assigned charges so as to also satisfy R2. The following lemma will be useful for this purpose, as well as later when we examine the hierarchical structure of valid tilings:

Lemma 3.6. Suppose that a tiling $T$ satisfies $\boldsymbol{R} \mathbf{1}$ and has charges assigned consistently across $\mathbf{R} 1$-edges. Then $T$ is valid if and only if the following charge transfer property holds:

(1) If $E_{1} \dashv^{N} E_{2}$, then $\operatorname{ch}\left(E_{1}\right)=\operatorname{ch}\left(E_{2}\right)$.

(2) If $E_{1} \dashv^{F} E_{2}$, then $\operatorname{ch}\left(E_{1}\right)=\operatorname{ch}\left(E_{2}\right)^{*}$.

Proof. By Lemma 2.1, a valid tiling satisfies the charge transfer property. So suppose, conversely, that $T$ satisfies R1 and has charges applied consistently across edges, and that the charge transfer property holds. We must show that $\mathbf{R 2}$ is also satisfied. Tiles can meet in one of three ways at an edge - meeting either two, one, or zero straight R1-segments:

(1) If an edge meets two straight line segments (left of Figure 7), then R2 is satisfied at this edge, since charges on R1-edges are consistent.

(2) If a straight line meets a turn over an edge, the turning tile is at either the terminus or the origin of the R1-line. In the former case (middle of Figure 7), there are no requirements for consistency of $\mathbf{R 2}$. The latter case is depicted in Figure 3; by Lemma 2.1, consistency is guaranteed by the charge transfer property.

(3) If two turns meet at the edge, $\mathbf{R 2}$ does not impose any restrictions, as seen on the right of Figure 7. 

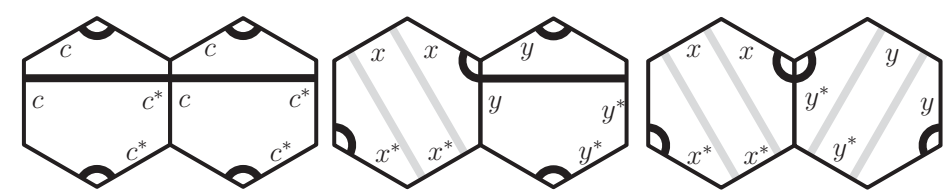

Figure 7. Consistency of charges splits into three cases in the proof of Lemma 3.6: when two straight R1-segments meet (left), when a straight segment meets a turn (middle), and when two turns meet (right). The two remaining cases of a straight segment meeting a turn are as in Figure 3.

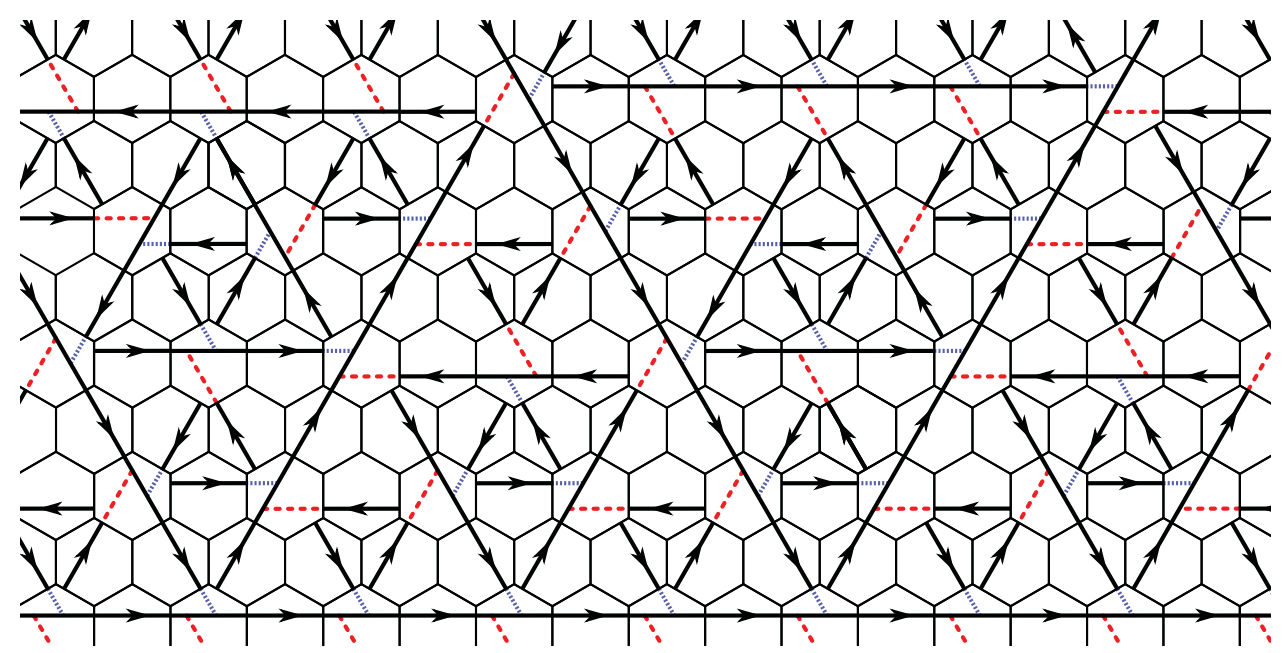

Figure 8. The R1-edge graph. Extensions of an edge $E_{1}$ to $E_{2}$ with $E_{1} \dashv^{N} E_{2}$ are given by dashed blue lines, and those with $E_{1} \dashv^{F} E_{2}$ are dotted red lines.

It follows that $T$ satisfies $\mathbf{R 2}$ and hence is valid, as required.

Given a tiling satisfying R1, we construct an infinite directed graph $G$, called the $\boldsymbol{R} \mathbf{1}$ edge graph, from the $\mathbf{R} 1$-edges by removing the turns, retaining orientations on $\mathbf{R} 1-$ edges, and extending $E_{1}$ forward to meet $E_{2}$ whenever $E_{1} \dashv E_{2}$ (see Figure 8). We note that each path component of this graph has exactly the tree structure defined by the growth condition of the tilings in [13]. Indeed, every valid tiling in [13] defines a valid tiling under the rules here using the R1-edge graph. However, the rules defined in [13] are not local in the sense of matching rules, and do not lead to a compact hull of tilings as they do in this paper (see $\S 4)$.

Lemma 3.7. An R1-tiling whose $\boldsymbol{R} 1$-edge graph $G$ has no loops and $\ell$ connected components may be assigned charges in precisely $2^{\ell}$ different ways so as to give a valid tiling.

Proof. For each component of $G$, choose any R1-edge and assign it one of the two possible charges. The charge transfer property forces the charges of the remaining edges of this component. Moreover, since this component is a tree, we cannot encounter inconsistencies 
in doing this. It follows that we may consistently apply charges R1-edges in two ways to this component so as to satisfy the charge transfer property. Repeating for each component gives a consistent tiling by Lemma 3.6.

The R1-edge graph $G$ of any tiling $T \in \Omega_{\mathrm{a}}$ has no loops. To see this, note that in any standard patch $P_{n}$, for any two (necessarily finite) edges $E_{1}$ and $E_{2}$ with $E_{1} \dashv E_{2}$ we have that $L\left(E_{1}\right)<L\left(E_{2}\right)$; this can be shown by induction, since $P_{n}$ is formed by embedding into the outer triangle of $P_{n}$ a copy of $P_{n-1}$ and three copies of its interior tiles. So the limiting tilings of $\Omega_{\mathrm{a}}$ also have this property. Hence, since a directed path in $G$ can only take one from edges to longer edges, there can be no loops, and by the foregoing may be consistently assigned charges to make valid tilings.

Combining this existence result with Theorem 2.4 proves Theorem 1.1, the main result of the paper.

\section{The hull of tilings}

In this section we explain the structure of the R1-triangles in valid tilings, which will allow us to classify the set of all valid tilings. We denote the set of all valid tilings by $\Omega$. This set carries a natural topology (see [19] for an introduction to the topological study of aperiodic tilings). The space $\Omega$ is sometimes called the (continuous) hull. Belonging to the hull is a local (in fact, edge-to-edge) condition, and as a consequence $\Omega$ is a compact space.

The rule $\mathbf{R} 1$ alone limits the possible sizes of the $\mathbf{R} 1$-triangles and the structure of $\mathbf{R} 1$-triangles inside of them. For $n \in \mathbb{N}$ we define $a(n)=2^{i}$, where $i \in \mathbb{N}_{0}$ is chosen as large as possible with $2^{i}$ dividing $n$. Recall that for an R1-triangle $\Delta$ we denote its size by $s(\Delta)=L(E)+1$, where $L(E)$ is the length of any of its R1-edges.

Proposition 4.1. Suppose a tiling $T$ satisfying $\boldsymbol{R} \mathbf{1}$ is given. For any $\boldsymbol{R} \mathbf{1}$-triangles $\boldsymbol{\Delta}$ in $T$, we have $s(\Delta)=2^{n}$ for some $n \in \mathbb{N} \cup\{0\}$. Moreover, suppose that $E_{1}$ and $E_{2}$ are $\boldsymbol{R} 1$-edges leading to or from a tile $t$ and, without loss of generality, are positioned so that $E_{1}$ is horizontal and $E_{2}$ extends up and to the right from $t$ (see Figure 9). Suppose that there are at least $k$ tiles from the right of (and not including) $t$ along $E_{1}$, and similarly $k$ tiles from $t$ up and to the right along $E_{2}$. Consider the $\boldsymbol{R} 1$-triangles $\Delta_{1}, \Delta_{2}, \ldots$, which share a tile with $E_{1}$, naturally ordered by where they meet $E_{1}$ from left to right. Then $s\left(\Delta_{n}\right)=a(n)$ for all $n=1, \ldots, k$.

Proof. Beginning at the left, for any $k$ we have $s\left(\Delta_{1}\right)=1$, since the two turns of $\Delta_{1}$ in $E_{1}$ and $E_{2}$ are already connected without straight edges. Suppose now that the previous result on the sizes $s\left(\Delta_{i}\right)$ holds for all $k<N$, and that for all R1-triangles $\Delta$ with $s(\Delta)<N$ we have $s(\Delta)=2^{n}$ for some $n \in \mathbb{N} \cup\{0\}$.

We claim that if there is a triangle of size $N$, then $N$ is a power of 2 . Indeed, let $n \in \mathbb{N}$ be such that $2^{n-1}<N \leq 2^{n}$. Take two edges $E_{1}$ and $E_{2}$ of $\Delta$ and note that they are positioned as in the statement of the proposition, so by induction we have that $s\left(\Delta_{2^{n-1}}\right)=2^{n-1}$. We see that $\Delta_{2^{n-1}}$ will only fit inside of $\Delta$ if $s(\Delta) \geq 2^{n}$, hence $s(\Delta)=2^{n}$, as required.

Next we show that for $E_{1}$ and $E_{2}$ as in the statement of the proposition, $s\left(\Delta_{N}\right)=a(N)$. Take $n \in \mathbb{N}$ such that $2^{n-1}<N \leq 2^{n}$. Let us write $t_{0}=t$, and $t_{i}$ the $i$ th tile from $t$ along 


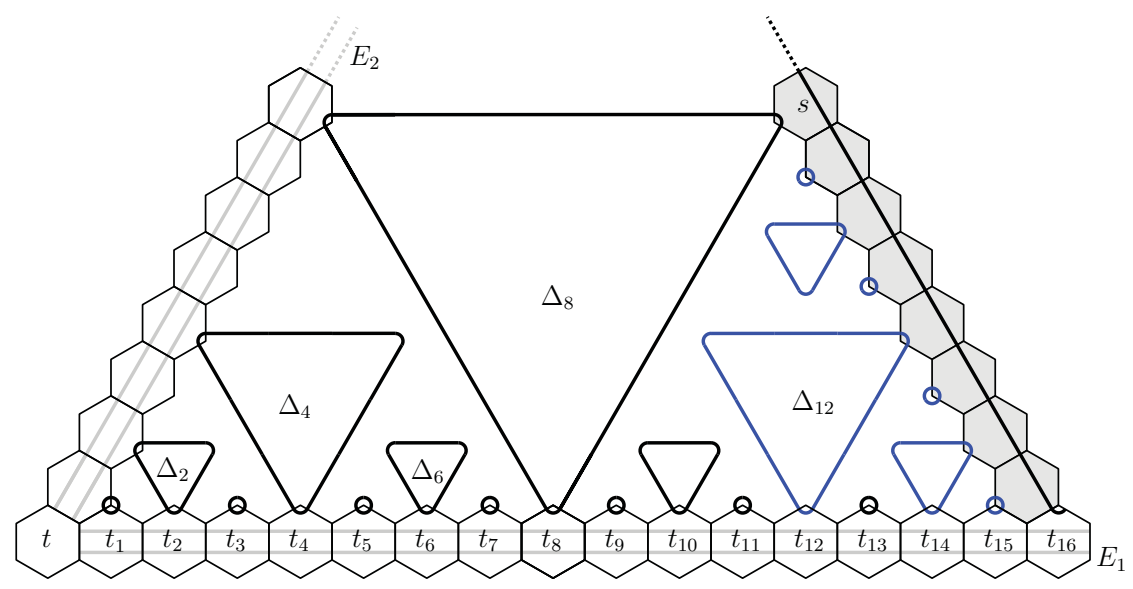

Figure 9. Proof of Proposition 4.1, for $k=16$ (the 16 tiles up and to the right of $t$ are not all indicated). The R1-edges to the right and upper right of $t$ are allowed to be offset in two possible ways, so these lines are marked in gray. Notice that the sizes of the triangles are, respectively, 1, 2, 1, 4, 1, 2, 1, 8, 1, 2, $1,4,1,2,1$ and finally $s\left(\Delta_{16}\right)=16$. Induction using the right edge of $\Delta_{8}$ and the edge between $t_{8}$ and $t_{16}$ forces the blue triangles, which force the line meeting $t_{16}$.

$E_{1}$ - that is, the tile shared with $\Delta_{i}$. If $N<2^{n}$, then by induction on the edge $E_{1}$ and the right-hand edge of $\Delta_{2^{n-1}}$ we see that $s\left(\Delta_{N}\right)=a\left(N-2^{n-1}\right)=a(N)$, as required. Indeed, the tile $t_{N}$ is a distance $N-2^{n-1}<2^{n-1}$ from $t_{2^{n-1}}$, and $s\left(\Delta_{2^{n-1}}\right)=2^{n-1}$, so the right-hand side of $\Delta_{2^{n-1}}$ is long enough to force $\Delta_{N}$.

Finally, suppose that $N=2^{n}$; we wish to show that $s\left(\Delta_{N}\right)=2^{n}$. Consider the collection $R$ of tiles heading in a straight line from $t_{N}$ up and to the left, terminating at $E_{2}$ (those in view in Figure 9 are shaded in gray, with $N=16$ ). Notice that the middle tile $s \in R$ contains the top right turn of $\Delta_{2^{n-1}}$, so the straight R1-segment of this $s$ is parallel to the row of tiles $R .{ }^{1}$ Similarly, the triangle $\Delta_{\left(2^{n-1}+2^{n-2}\right)}$ (given by $\Delta_{12}$ in Figure 9) has its top right corner in a tile of $R$ lying halfway between $t_{N}$ and $s$, so the straight $\mathbf{R} 1$-segment of this tile is also parallel to $R$. We may repeat this to see that each tile in $R$ between $s$ and $t_{N}$ has a straight-line segment running parallel to the direction of $R$, so these segments must be contiguous and form part of an R1-edge running at least between $s$ and $t_{N}$. This R1-line is already composed of $2^{n-1}$ straight sections, and so by the first part of the proof, restricting the sizes of triangles, it must in fact be of length at least $2^{n}-1$ (alternatively, we could repeat the previous argument on the triangular region between $E_{2}$ and the top edge of $\Delta_{2^{n-1}}$ ). It cannot be longer, or else it would pass through $E_{1}$ or $E_{2}$, so we conclude that $s\left(\Delta_{N}\right)=2^{n}=a(N)$, as required.

${ }^{1}$ Notice that if $\mathbf{R 2}$ is also satisfied, we may conclude already that the R1-edge $E$ passing through $s$ has length at least $L(E)>2^{n-1}$, by Lemma 2.2, and so extends all the way to $t_{N}$. Since there are no triangles of size between $2^{n-1}$ and $2^{n}$, by induction, we may thus already conclude that $s\left(\Delta_{N}\right)=N$. However, as is shown, R2 is not needed here. 
Notice that for $k=2^{n}$ we have $s\left(\Delta_{k / 2}\right)=k / 2$. So for any R1-triangle (or more generally, a triangular region bounded by $\mathbf{R} 1$-edges), Proposition 4.1 shows that $\mathbf{R} 1$ alone forces an $\mathbf{R} 1$-triangle of half the size positioned with corners at the midpoint of the bounding edges. So the hierarchical pattern of triangles given in constructing the standard patterns in $\S 3$ is forced by $\mathbf{R} \mathbf{1}$.

Given a tiling $T \in \Omega$, let $\mathbf{R} \mathbf{1}(T)$ be the associated $\boldsymbol{R} 1$-tiling given by forgetting the $\mathbf{R 2}$ decorations. Similarly, we let $\mathbf{R} \mathbf{1}(\Omega)=\{\mathbf{R} \mathbf{1}(T): T \in \Omega\}$. We now determine which tilings can belong to $\mathbf{R} \mathbf{1}(\Omega)$, which can be split into the following classes:

(1) There is no infinite R1-line and

(a) every $\mathbf{R} 1$-triangle is contained in infinitely many others or

(b) every $\mathbf{R} \mathbf{1}$-triangle is contained in only finitely many others.

(2) There is at least one infinite R1-line and

(a) there is an infinite R1-line containing no turn or

(b) every infinite R1-line contains a turn.

Recall from Definition 3.4 that $\Omega_{\mathrm{a}}$ is the collection of tilings whose finite patches are contained in translates of the standard patches $P_{n}$ given in $\S 3$.

Lemma 4.2. If $T \in \boldsymbol{R} \mathbf{1}(\Omega)$ is in class $1 a$, then $T \in \Omega_{a}$.

Proof. Since every triangle is contained in a larger one, we may construct an infinite nested sequence of triangles $\Delta_{1}, \Delta_{2}, \ldots$ whose interiors cover the entire plane. By Proposition 4.1, the interiors of these triangles are forced to have standard R1decorations, so $T \in \Omega_{\mathrm{a}}$, as required.

Lemma 4.3. If $T \in \boldsymbol{R} \mathbf{1}(\Omega)$ is in class $1 b$, then $T \in \Omega_{a}$.

Proof. Consider an 'outer R1-triangle' $\Delta$ of $T$ - that is, one which is not contained in any other triangle. Take an edge $E_{1}$ of $\Delta$ and follow the sequence of edges $E_{1} \dashv E_{2} \dashv$ $E_{3} \dashv \cdots$. Since the triangle containing $E_{k}$ is exterior to that containing $E_{k+1}$, we have that $E_{k} \dashv^{F} E_{k+1}$ for all $k$. Analogously to the proof of Lemma 2.2, we thus construct a spiral of edges whose triangles are all exterior to each other. Lemma 2.2 implies that the edges become longer, infinitely spiraling outward. Since all triangles are exterior to each other, we see that arbitrarily large patches about the initial edge $E_{1}$ are contained in the triangular regions bounded by $E_{k}$ and $E_{k+1}$. But the pattern of R1-triangles in such regions are forced to be standard ones also found between edges of $\mathbf{R} 1$-triangles, by Proposition 4.1. So arbitrarily large patches about $E_{1}$ are patches of tilings of $\Omega_{\mathrm{a}}$, so $T \in \Omega_{\mathrm{a}}$, as required.

In summary, those generic tilings $T \in \mathbf{R} \mathbf{1}(\Omega)$ without an infinite $\mathbf{R} 1$-line are also in $\Omega_{\mathrm{a}}$. By Lemma 3.7 and the remarks following it, we may choose compatible R2-decorations for such tilings to give valid tilings. The edge graph is typically, but not always, connected (see Lemma 6.4).

Lemma 4.4. Suppose that $T \in \boldsymbol{R} \mathbf{1}(\Omega)$ is in class 2a. Let $L$ be the unique infinite $\boldsymbol{R} \mathbf{1 -}$ line without turn. Then $T$ is a union of two partial tilings $T_{1}$ and $T_{2}$, where $T_{1}$ covers 
a half-plane one side of $L$ and $T_{2}$ the other, and where each $T_{i}$ is a subset of tiles from some $T_{i}^{\prime} \in \Omega_{a}$.

Proof. Consider the pattern of R1-triangles one side of $L$, and take any triangle $\Delta$ in it with turn on a tile carrying $L$. The right-hand edge of $\Delta=\Delta_{1}$ leads to the left-hand edge of another triangle $\Delta_{2}$, which also shares a turn with $L$ by Lemma 2.2. Repeating this, we construct a sequence of triangles $\left(\Delta_{i}\right)_{i \in \mathbb{N}}$ which meet tiles of $L$. Note that by Lemma 2.2 and Proposition 4.1, we have $s\left(\Delta_{i+1}\right) \geq 2 s\left(\Delta_{i}\right)$, and then the triangles $\Delta_{i}$ force standard R1-decorations in increasingly large regions in the half-plane. More precisely, the union of the inside of $\Delta_{i}$, the triangular region between its left edge and $L$, and the other triangular region between its right-edge and $L$ is forced to be a standard decoration seen in some $P_{n}$. For these forced standard patches, either they cover the whole half-space or $s\left(\Delta_{i+1}\right)=2 s\left(\Delta_{i}\right)$ for sufficiently large $i$. In the latter case, an analogous argument to that in the final paragraph of the proof of Proposition 4.1 implies the existence of an infinite R1-triangle meeting $L$ to the left of $\Delta$. Again by Proposition 4.1, this forces a standard R1-decoration on this half-plane.

For a tiling $T$ as before with $T \notin \Omega_{\mathrm{a}}$, we call $T$ a tiling with an infinite fault line. For tilings $T \in \Omega_{\mathrm{a}}$, a triangle meeting $L$ has the same size as the triangle opposite it across $L$. But since our matching rules are edge-to-edge, one may freely shift the tiles of one halfplane relative to the other half. Again, by Lemma 3.7, any such tiling can be equipped with compatible $\mathbf{R 2}$-decorations and so is an element of $\mathbf{R} \mathbf{1}(\Omega)$.

Finally, suppose that we are in Case 2b. Consider the infinite R1-edges with turn $L_{i}$. By Lemma 2.2, the edge of each $L_{i}$ heading toward the turn leads to another infinite edge. Then the only possibility is that there are three infinite $\mathbf{R} 1$-edges $L_{1}, L_{2}$, and $L_{3}$ arranged in a cyclic fashion as in Figure 10. Let $E_{i}$ be the edge of $L_{i}$ leading to the turn and $E_{i}^{\prime}$ be the other edge of $L_{i}$. There are two cases: either $E_{i} \dashv^{F} E_{i+1}$ or $E_{i} \dashv^{F} E_{i+1}^{\prime}$ for all $i$ (considered modulo 3 , and with the $L_{i}$ ordered appropriately). The first case (left of Figure 10) is ruled out by Lemma 2.1. The second possibility (right of Figure 10), however,
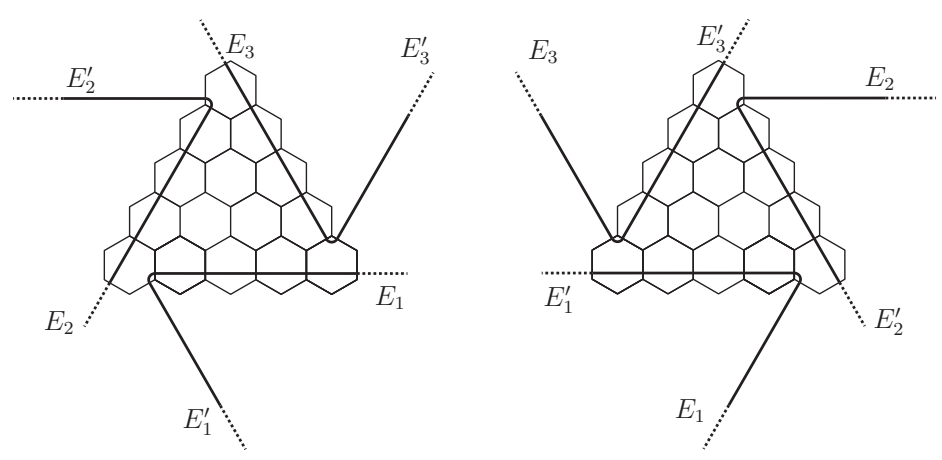

Figure 10. In case $2 \mathrm{~b}$, the three infinite lines with turns are forced to arrange themselves as a cycle. The left-hand picture shows a configuration which cannot occur. The right-hand picture, in this case an infinite 1-cycle, can occur. 
is possible. By Proposition 4.1, the pattern of R1-triangles is completely determined by the number of tiles of $E_{i}^{\prime}$ starting from the tile after that containing the turn of $L_{i}$ up to and including the tile containing the turning tile of $E_{i-1}$, and there are $2^{n+1}$ such tiles for some $n \in \mathbb{N} \cup\{0\}$. We call such a tiling an infinite $n$-cycle. Up to translation, for each $n \in \mathbb{N}$ there are precisely two such R1-tilings (which are related by a rotation) by Proposition 4.1. We have thus proved the following:

Theorem 4.5. If $T \in \boldsymbol{R} 1(\Omega)$, then one of the following is true:

(1) $T \in \Omega_{a}$.

(2) $T$ is a tiling with infinite fault line.

(3) $T$ is an infinite $n$-cycle tiling.

In each case, there are $2^{\ell}$ tilings $T^{\prime} \in \Omega$ with $\boldsymbol{R} \mathbf{1}\left(T^{\prime}\right)=T$, where $\ell$ is the number of path components of the $\boldsymbol{R} 1$-edge graph of $T$.

Generically, $\ell=1$, but there are also cases where $\ell=2$ or 3 in Theorem 4.5(1) (see Lemma 6.4 ). In case $2, \ell=1,2$, or 3 , depending on whether there are, respectively, 0,1 , or 2 infinite triangles meeting the fault line. In case $3, \ell=3$.

\section{Generating valid tilings by substitution}

In this section we build a primitive and recognizable substitution rule which generates valid tilings. This will allow us to analyze frequencies of patches and deduce the unique ergodicity of the hull of all valid tilings. Measure-theoretically, almost all valid tilings will belong to the minimal component of substitution tilings.

\subsection{MLD triangle tilings}

For a 'stone inflation' [2], inflates of tiles are precisely tiled by copies of the originals. Since one may not tile the hexagon with smaller hexagons, we cannot define a stone inflation for our original tiles, although one may define a 'substitution with overlaps' (like the Penrose kite-and-dart or rhomb substitution [15]) or a 'pseudo-inflation' [1, 2].

We choose instead to pass to the dual triangle tiling, which will allow us to define a stone inflation that generates tilings that are MLD to valid tilings. Hence our prototile set will consist of decorated equilateral triangles. Each triangle has edges labeled with

- an arrow at each edge's center, pointing in one of two possible directions perpendicular to the edge, and

- a charge, either + or - .

So each triangle edge has one of four possible labelings. The direction is assigned by the direction of offset toward the R1-line which crosses the corresponding hex-tile edge (see Figure 11). If this arrow is pointed toward a straight section of an R1-edge, we assign it the same charge as this edge. Otherwise, the arrow is pointed toward the start of a left turn, for which we may determine a charge analogously to the charge transfer property of Lemma 3.6. That is, if the turn $t$ is part of a hex-tile with straight R1-segment $s$ 


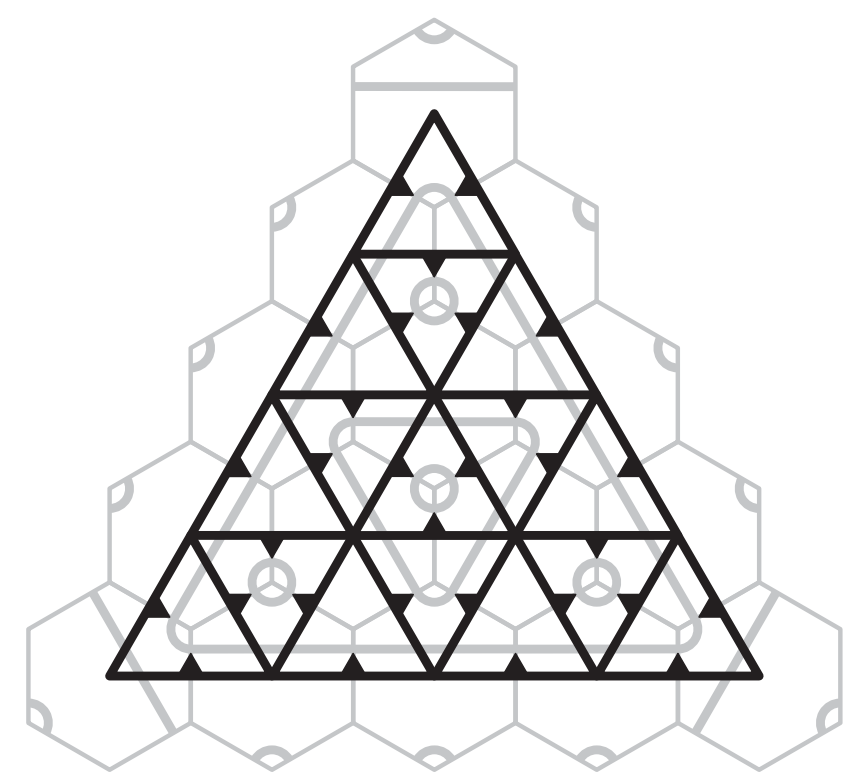

Figure 11. The MLD equivalence on small patches of a valid tiling and a tiling admitted by $\varphi$, with charges removed for clarity.

of charge $c$, then we also label our triangle edge with charge $c$ if $s$ is offset near to $t$. Otherwise, if $s$ is offset far from $t$, we assign our triangle edge charge $c^{*}$.

It is easily seen that a valid tiling by the tile of Figure 1 determines, by a locally defined rule, a triangle tiling and vice versa, so two such are MLD. Unless stated otherwise, we assume in this section that all tilings have been converted to triangle tilings, and call such tilings valid if their corresponding hex tilings are.

\subsection{The substitution}

Given a decoration of R1-triangles from a valid tiling, there is a natural way of associating to it another whose level $n \geq 1$ triangles correspond to those of level $(n-1)$ from the original. Simply double the size of each triangle, preserving the charges of their edges, and add new level 0 triangles in the gaps. By Lemma 3.6, this gives the R1-decorations of another valid tiling. The procedure defines our stone inflation on triangle tiles: each triangle is inflated by a factor of 2 and split into four triangles of the original size, the labels on the outside edges are preserved, and the central triangle has all edges directed inward (which corresponds to adding a level 0 triangle). The charges of the internal edges are the unique ones which satisfy the charge transfer property of Lemma 3.6 upon further substitutions, or equivalently, how charges are assigned in the MLD relation already described. In the triangle tilings, a straight section of edges labeled with the same directions corresponds to an $\mathbf{R} 1$-edge, whereas a vertex of a triangle between two inward-pointing edges corresponds to an R1-turn. 


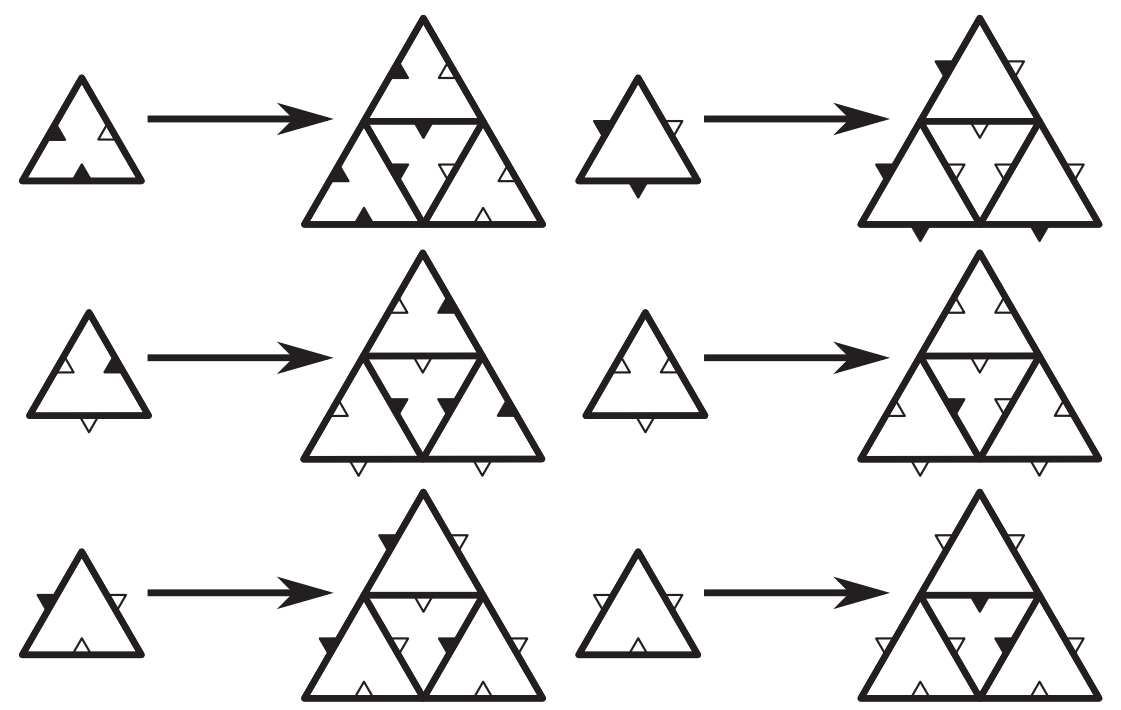

Figure 12. The tiling substitution $\varphi$. Positively charged edges are indicated with black arrows and negatively charged edges with white arrows.

This defines a tiling substitution $\varphi$ of finite local complexity [2] on the set of all edgelabeled triangles (see Figure 12). As usual, we may extend $\varphi$ to act on (possibly infinite) patches. Recall that $\varphi$ is called primitive if there exists some $N \in \mathbb{N}$ such that $\varphi^{N}(t)$ contains a copy of each tile for any prototile $t$. A primitive substitution rule is defined by the six prototiles in Figure 12, along with their rotates and charge-flips. Since each tile is asymmetric, this gives a prototile set $\mathcal{P}$ of $6 \times 6 \times 2=72$ tiles.

A tiling is said to be admitted by the substitution $\varphi$ if every finite patch is contained in a translate of an $n$-supertile, which is a patch $\varphi^{n}(p)$ for some $n \in \mathbb{N}$ and $p \in \mathcal{P}$. We also refer to a 1 -supertile as simply a supertile. We denote by $\Omega_{\varphi}$ the set of all tilings admitted by $\varphi$.

For tilings $T$ and $T^{\prime}$ with $T=\varphi^{n}\left(T^{\prime}\right)$, we call $T^{\prime}$ a level-n supertiling of $T$, and a tile $t \in T^{\prime}$ a level- $n$ supertile. By primitivity, tilings admitted by $\varphi$ exist and have admitted supertilings of all levels. When the supertiling is always uniquely defined by $T$ (and then necessarily MLD to it), we call $\varphi$ recognizable.

Lemma 5.1. The substitution $\varphi$ on the prototile set $\mathcal{P}$ is primitive and recognizable.

Proof. Primitivity may be checked by hand, or more quickly by a computer. ${ }^{2}$ Let $T$ be an admitted tiling, with associated supertiling $T^{\prime}$ (which we scale here by a factor of 2 ). Notice that under substitution, only the central triangle of a supertile has all edges directed inward. So the tiles of $T^{\prime}$ are located periodically, with centers at precisely the locations of the all-inward-directed triangles of $T$ (which correspond to R1-triangles of

\footnotetext{
${ }^{2}$ We thank Franz Gähler for assisting us with this.
} 
size 0). There is also no choice for the labelings on the exteriors of supertiles; they must agree with the edges they overlap in $T$, since labels of exterior edges are kept constant under substitution. So the supertiling $T^{\prime}$ is locally determined and uniquely defined, meaning that $\varphi$ is recognizable.

Lemma 5.2. A tiling admitted by $\varphi$ is valid - that is $\Omega_{\varphi} \subset \Omega$.

Proof. By the definition of the substitution rule, an $n$-supertile $\varphi^{n}(p)$, for $p \in \mathcal{P}$, has charges assigned consistently to R1-edges (since edges of length $2^{n}$ are doubled in length under substitution and given the same charge). The charge transfer property of $\S 3.2$ also holds, by the way that internal edges are assigned charges in the definition of $\varphi$. Since a tiling admitted by $\varphi$ can be covered by such patches, the corresponding hex tiling is valid, by Lemma 3.6.

\subsection{Supertile decompositions of valid tilings}

Not all valid tilings in $\Omega$ are admitted by $\varphi$. For example, tilings containing $n$-cycles or fault lines are not. However, we shall see that every valid tiling has some form of decomposition into supertiles of all levels. This will allow us to deduce that all valid tilings have uniform patch frequencies, even if they are not admitted by $\varphi$.

Recall that in defining the tiling substitution $\varphi$, we kept only 72 of the $2 \times\left(4^{3}\right)=128$ possible decorated triangles for our prototile set. We omit precisely the tiles of Figure 13 , along with their rotates and charge-flips. The first two are preserved under rotation by $2 \pi / 6$, so together account for eight tiles. The third class is asymmetric and may have right-hand direction and charge assigned freely, so these account for the remaining $2 \times 4 \times 6=48$ tiles. We define $\mathcal{P}^{\prime}$ to be the union of prototiles $\mathcal{P}$ and the eight tiles in the first and second classes of Figure 13. Then $\varphi$ also defines a (nonprimitive) substitution on $\mathcal{P}^{\prime}$.

Lemma 5.3. A triangular region $P$ of a valid tiling bounded between three $\boldsymbol{R} \mathbf{1}$-edges is given by $\varphi^{n}(t)$ for $t \in \mathcal{P}^{\prime}$.

Proof. The three outer edges of $P$ are consistently directed and charged. Let $t$ be the triangular tile with corresponding labels of its edges. Then $t$ cannot belong to the third class of tile in Figure 13. Indeed, in that case the left edge necessarily has to
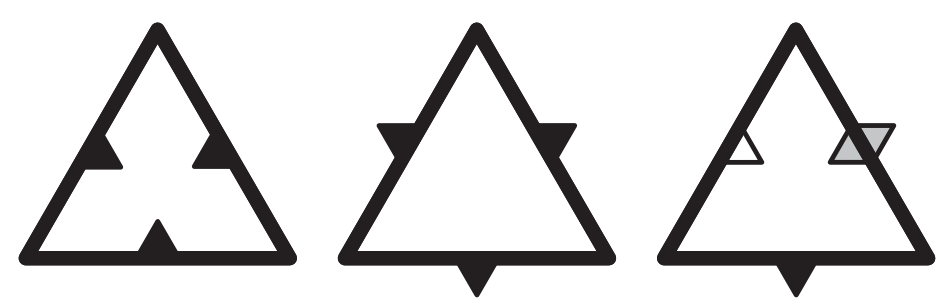

Figure 13. Tiles omitted from the prototile set $\mathcal{P}$ are given by these three classes, where we take all charge-flips and rotates. The third class may have its right-hand edge labeled freely. 
continue downward from $P$; it cannot turn toward the bottom edge, since that is directed downward. This then violates the charge transfer property with the bottom edge.

By Proposition 4.1, the interior of $P$ has R1-decorations determined by the size of $P$, which must have edge lengths a power of 2 . So the R1-decorations of $P$ agree with $\varphi^{n}(t)$. By construction, the outer edges of $P$ have charges agreeing with $\varphi^{n}(t)$. Charges of interior R1-edges of $P$ are determined by the charge transfer property of Lemma 3.6. By the construction of $\varphi$, the charge transfer property holds on $\varphi^{n}(t)$ too, so the two patches agree.

The next proposition exhibits the extent to which valid tilings may be equipped with supertile decompositions. There is an interesting singular case, which has an analogous occurrence in the Socolar-Taylor tilings. Notice that a rotated copy of $P_{n}$ may be found at the center of $P_{n+1}$ (see Figure 6). So there is an R1-tiling, with global 3-fold rotational symmetry, given as the union $P_{1} \cup r\left(P_{2}\right) \cup P_{3} \cup r\left(P_{4}\right) \cup \cdots$, where $r$ represents rotation by $2 \pi / 6$ and each patch has common center over the origin. A rigid motion of this is called a $P_{\infty}$ tiling; in [11] it is called an iCW-L tiling, short for infinite concurrent w-line tiling.

Proposition 5.4. Let $T$ be a valid tiling. Then we have the following:

(1) If the $\boldsymbol{R} 1$-decorations of $T$ are a rigid motion of $P_{\infty}$, then $T$ has a unique and locally defined level-n supertiling for each $n \in \mathbb{N}$ with prototiles in $\mathcal{P}$, or possibly also one occurrence at each level of the tile in the first class of Figure 13.

(2) If $T$ is an $n$-cycle, then the infinite $\boldsymbol{R} \mathbf{1}$-edges bound seven regions, one of which is a translate of $\varphi^{n}(t)$, where $t \in \mathcal{P}$ or the second class of Figure 13, and the other six of which have unique supertile decompositions with prototile set $\mathcal{P}$.

(3) If $T$ has an infinite $\boldsymbol{R} 1$-line without turn $L$, then the infinite patches between infinite $\boldsymbol{R} \mathbf{1}$-edges appear in certain tilings admitted by $\varphi$ with prototile set $\mathcal{P}$.

(4) Otherwise, $T$ is admitted by $\varphi$ with prototile set $\mathcal{P}$.

Proof. Case (1): There are R1-triangles $\Delta_{1}, \Delta_{2}, \ldots$ with identical centers and $s\left(\Delta_{n}\right)=2^{n}$ for all $n \in \mathbb{N}$. By Lemma 5.3, the patch of tiles in each $\Delta_{n}$ is given by $\varphi^{n}(t)$, where $t$ is an all-inward-directed triangle with outer charges given by the charges of the edges of $\Delta_{n}$. The existence of unique and locally defined level- $n$ supertilings then follows in the same way as the proof of Lemma 5.1. Notice that once the charges for $\Delta_{1}$ are chosen, the charges for the remaining triangles are determined, since each edge of $\Delta_{n+1}$ is connected to precisely one edge of $\Delta_{n}$ in the R1-edge graph (more precisely, we rotate the charges $2 \pi / 6$ counterclockwise between each triangle). In particular, if any $\Delta_{k}$ is chosen with all edges having the same charge, then all other $\Delta_{n}$ have only this charge too. A tile corresponding to a single-charge level 1 triangle does not appear as the substitute of a tile from $\mathcal{P}$, and only occurs once (at the center, rotated, of a substitute of itself), so it occurs precisely once at each level of supertiling.

Case (2): By Lemma 5.3, the finite region bounded between the infinite R1-edges is given by $\varphi^{n}(t)$, where $t$ is a triangle with all edges directed outward. The six infinite regions bounded between infinite R1-edges are infinite unions of triangular regions of size $2^{n}$, for $n \in \mathbb{N}$, bounded between R1-edges and with R1-decorations determined by 
Proposition 4.1, which by Lemma 5.3 can be expressed as an infinite nested union of $n$-supertiles $\varphi^{n}(t)$ with a common corner at the intersection of R1-edges. In fact, the edges of $\varphi^{n}(t)$ overlapping with the infinite R1-edges must have the same corresponding charges, and the remaining edge has charge determined by the transfer property, so is a tile in $\mathcal{P}$.

Case (3): Take a half-plane on one side of $L$. If there are no other infinite R1-edges in this region, it can be covered by supertiles $\varphi^{n}(t)$ for $t \in \mathcal{P}$ which share an outer edge with $L$, by Proposition 4.1 and Lemma 5.3. Again, each $t \in \mathcal{P}$ by the charge transfer property. Otherwise, there is an infinite R1-line with a turn in this half-plane, which is therefore decomposed into three regions with supertile decompositions analogous to the six infinite regions of the previous case. Repeating for the other half-plane, we see that there is a unique covering of $T$ by $n$-supertiles for each $n \in \mathbb{N}$.

Case (4): If none of the other cases apply, Proposition 4.1 implies that there is an infinite nested sequence $\Delta_{1}, \Delta_{2}, \ldots$ of triangular regions $\Delta_{n}$ bounded between $\mathbf{R} 1$-edges with sides of length $2^{n}$ and whose union covers $\mathbb{R}^{2}$ (if it did not, then this would lead to an infinite R1-edge). By Lemma 5.3 these triangles are given by $\varphi^{n}\left(t_{n}\right)$ for $t_{n} \in \mathcal{P}^{\prime}$. However, $\Delta_{n}$ appears off-center in $\Delta_{n+1}$ infinitely often, since $T$ is not a $P_{\infty}$ tiling. Since the second class of tile of Figure 13 cannot appear in a substituted tile, and the first class appears only at the center of a substitute of itself, each $t_{n} \in \mathcal{P}$, so $T$ is admitted by $\varphi$.

Remark 5.5. The foregoing shows that valid tilings have unique supertile decompositions of all levels, except for possibly the $P_{\infty}$ tilings (which are either admitted by $\varphi$ or admitted by extending to a nonprimitive prototile set) and $n$-cycle tilings (which have supertile decompositions of all levels in six infinite regions, covering the whole plane except for a central level- $n$ supertile). This proof shows that the bad tiles, given as the first two classes of Figure 13, may occur as follows:

(1) The first class (an R1-triangle with only one charge of edge) can only be part of a $P_{\infty}$ tiling or $n$-cycle. For the $P_{\infty}$ tiling, either the tiling is admitted or there is precisely one such bad tile at each level, which appears at the center of the next one. This is similar to the situation for the Socolar-Taylor tiling, where the existence of a level 1 R1-triangle forming a vertex of a single color forces a $P_{\infty}$ tiling and the remaining decorations. Just as for the Socolar-Taylor tile, there are eight possible ways of decorating a $P_{\infty}$ tiling to get a valid tiling, with six of them admitted by the substitution and the remaining two 'defect' tilings having 3-fold rotational symmetry.

(2) The second class of bad tiles of Figure 13 may occur in only one role: as a level$n$ triangle of an $n$-cycle tiling when this tiling has 3 -fold symmetry. In this case, precisely one bad tile occurs at the center of the next up to level $n$.

\section{Ergodic and dynamical properties}

We now analyze some fundamental properties of the dynamical system associated to the hull of valid tilings and how they relate to the Socolar-Taylor and Penrose $\left(1+\epsilon+\epsilon^{2}\right)$ tilings. 


\subsection{Unique ergodicity}

The supertile decompositions will allow us to show that all patches in valid tilings appear with well-defined frequencies, from which we may deduce unique ergodicity of the tiling dynamical system. Again, we work with the triangular versions of our tilings.

Unique ergodicity of a tiling dynamical system given by the orbit closure of a tiling is equivalent to the tiling having uniform patch frequencies [2]. The hull of valid tilings is not the orbit closure of a single tiling. Indeed, $\Omega$ contains $n$-cycle and fault-line tilings (see Theorem 4.5), which contain 'defective' finite patches that do not appear in orbit closures of the others. However, we can still prove that the collection of valid tilings has uniform patch frequencies in the following sense:

Definition 6.1. We say that a collection $X$ of tilings has uniform patch frequencies if for each finite patch $P$, there exists some $\delta_{P} \geq 0$, its density, such that for all $\epsilon>0$ there exists $R>0$ such that

$$
\left|\frac{\#(P, T, R)}{V_{R}}-\delta_{P}\right|<\epsilon
$$

for any $T \in X$, where \#(P,T,R) is the number of occurrences of $P$ inside the ball of radius $R$ centered at the origin of $T$ and $V_{R}=\pi R^{2}$ is the area of an $R$-ball.

We scale our tilings so that the density of all tiles is equal to 1 . Then the densities of all possible patches of the same shape sum to 1 and we refer to these densities as patch frequencies. Some authors demand that frequencies be strictly positive in the definition of uniform patch frequencies, which implies that there is a unique invariant measure and that the open sets have strictly positive measure (that is, the system is strictly ergodic). In our case, there will exist patches with frequency 0 in the 'defect' tilings (i.e., the $n$-cycles, those with fault lines, or the $P_{\infty}$ tilings with 3 -fold symmetry).

The hull of tilings coming from a primitive, recognizable substitution rule always has uniform patch frequencies. Using this in conjunction with the result on supertile decompositions of valid tilings from Proposition 5.4 allows us to deduce uniform patch frequencies of all valid tilings:

Proposition 6.2. The collection $\Omega$ of valid tilings has uniform patch frequencies.

Proof. Take a patch $P$ and valid tiling $T$, which we normalize to have tiles of unit area. Then $P$ appears with frequency $\delta_{P}$ in any tiling of $\Omega_{\varphi}$ (where $\delta_{P}=0$ if it does not appear in any tiling of $\Omega_{\varphi}$ ). We claim that $P$ appears with frequency $\delta_{P}$ in $T$ too.

In Proposition 5.4(4) the tiling is already in $\Omega_{\varphi}$, so this is immediate. Cases (2) and (3) are also clear by the following argument. Take a large $k \in \mathbb{N}$ and remove from $T$ a neighborhood $N$ of the boundaries of $k$-supertiles, as well as the central triangle of an $n$-cycle in case (2), so that occurrences of $P$ in $\mathbb{R}^{2} \backslash N$ appear only in interiors of $k$-supertiles (which we know appear as patches in $\Omega_{\varphi}$ ). For a large $k$ and $R>0$, only a negligible proportion of any $R$-ball intersects $N$, and on $\mathbb{R}^{2} \backslash N$ occurrences of $P$ have the same frequency as in tilings of $\Omega_{\varphi}$. So we see that $P$ occurs in $T$ with this same frequency in $T$ too. 
Suppose then that $T$ is in case (1) of Proposition 5.4, and let $\epsilon_{1}, \epsilon_{2}>0$ be arbitrary. Choose a large integer $k \in \mathbb{N}$. Let $s$ be the smallest number of occurrences of $P$ inside any $k$-supertile $\varphi^{k}(t)$, for $t \in \mathcal{P}$. Similarly, let $S$ be the largest number of occurrences of $P$ in any level- $k$ supertile, where we include potential occurrences of $P$ which may intersect the boundary. By uniform patch frequencies in $\Omega_{\varphi}$, we may choose $k$ large enough so that

$$
2^{k}\left(\delta_{P}-\epsilon_{1}\right) \leq s \leq S \leq 2^{k}\left(\delta_{P}+\epsilon_{1}\right) .
$$

Next take some large $R>0$. Let $n$ be the smallest number of $k$-supertiles (which may also exclude the single 'bad' $k$-supertile which does not appear in $\Omega_{\varphi}$, as in Remark 5.5) that are contained in any $R$-ball, and $N$ be the largest number of $k$-supertiles which can intersect any $R$-ball. By making $R$ large enough relative to $2^{k}$, we can ensure that

$$
\frac{V_{R}}{2^{k}}\left(1-\epsilon_{2}\right) \leq n \leq N \leq \frac{V_{R}}{2^{k}}\left(1+\epsilon_{2}\right) .
$$

By counting occurrences of $P$ in interiors of $k$-supertiles or ones which may occur along boundaries of supertiles, we see that

$$
n \cdot s \leq \#(P, T, R) \leq N \cdot S+2^{k},
$$

where on the right-hand side we use the (crude) upper bound of $2^{k}$ possible occurrences of $P$ inside the single 'bad' $k$-supertile. Hence

$$
\left(1-\epsilon_{2}\right)\left(\delta_{P}-\epsilon_{1}\right) \leq \frac{\#(P, T, R)}{V_{R}} \leq\left(1+\epsilon_{2}\right)\left(\delta_{P}+\epsilon_{1}\right)+\frac{2^{k}}{V_{R}} .
$$

So by making $\epsilon_{1}$ and $\epsilon_{2}$ sufficiently small, and $R$ sufficiently large relative to $k$, we see that

$$
\left|\frac{\#(P, T, R)}{V_{R}}-\delta_{P}\right|
$$

can be made as small as desired.

An application of the ergodic theorem yields the following:

Corollary 6.3. The dynamical system $\left(\Omega, \mathbb{R}^{2}\right)$ is uniquely ergodic, and with respect to the unique invariant measure, almost every tiling of $\Omega$ is in $\Omega_{\varphi}$.

Proof. One may consider a transversal to the tiling flow on the continuous hull, which here is a $\mathbb{Z}^{2}$-shift. This is given, for example, by considering tilings where the origin lies at the center of a tile. This canonical transversal $X$ is a totally disconnected space whose clopen sets have measure determined by patch frequencies, which exist by the foregoing. Unique ergodicity of the continuous hull follows from that of its transversal.

Let $X_{r} \subset X$ denote the set of valid tilings with $r$-patch at the origin belonging, up to translation, to any substitution tiling (i.e., such a patch appears in a $k$-supertile for some $k \in \mathbb{N}$ ). Then each $X_{r}$ has measure 1 , as the frequencies of nonadmitted patches have frequency 0 (and so, as before, by the ergodic theorem have measure 0 in $\Omega$ ). Since $X_{\varphi}=\bigcap_{r>0} X_{r}$, continuity from above of the measure implies that $X_{\varphi}$ has full measure in $X$. The same then follows for the continuous hull. 


\subsection{Topological factors and spectral properties}

Since almost every valid tiling is in the minimal core $\Omega_{\varphi}$, for the spectral properties of our tilings it is equivalent to study this more well-behaved space instead, which we do for the remainder of the paper. We observe that there is an order 2 homeomorphism, equivariant with respect to the action of translation, on $\Omega_{\varphi}$ (and $\Omega$ ) given by charge-flip. It follows that $\left(\Omega_{\varphi}, \mathbb{R}^{2}\right)$ is not an almost-everywhere 1-to- 1 extension of its maximal equicontinuous factor, and so cannot have pure point dynamical spectrum [4] (or diffraction spectrum $[6,12])$. We also consider the quotient space by charge-flip, which we denote by $\Omega_{\varphi}^{ \pm}$. Its elements can be naturally viewed as tilings: we remove charge labelings at edges and replace them with labels for each triangle vertex which indicate whether or not the charge flips between the vertex's adjacent edges. It is easily seen that such tilings can be produced by a substitution rule, given by that of Figure 12 but with charge-flip pairs of tiles identified. This is a substitution of 36 tiles, six up to rotation equivalence.

The hull of all Socolar-Taylor tilings also has a minimal core generated by substitution, called the Taylor tilings in [1], which we denote by $\Omega_{\mathrm{ST}}$. We recall also from [1] the hulls of half-hex tilings $\Omega_{\mathrm{HH}}$, arrowed half-hex tilings $\Omega_{\mathrm{a}}$, and Penrose $\left(1+\epsilon+\epsilon^{2}\right)$-tilings $\Omega_{\epsilon}$. Finally, we let $\mathbb{S}_{2}^{2}$ be the 2-dimensional maximal equicontinuous factor (MEF) of these dynamical systems, which is given by the 2-dimensional dyadic solenoid.

Recall that the arrowed half-hex tilings of $\Omega_{\mathrm{a}}$ are MLD to standard $\boldsymbol{R} 1$-tilings, hex tilings with R1-decorations as appearing in the Socolar-Taylor tilings [1]. These tilings can be generated by the substitution rule of Figure 12, where charge decorations are removed. The half-hex tilings may be considered as such tilings where edges are not offset, but the locations and directions of R1-turns are known. Since the offset of an $\mathbf{R} 1$-edge is determined on any R1-triangle with a turn (as this determines the inside of a triangle), the map is 1-to-1 on tilings without a bi-infinite (straight) R1-edge. On the other hand, it is 2-to-1 on tilings with a bi-infinite R1-edge, since this may be offset in one of two directions to obtain a tiling of $\Omega_{\mathrm{a}}$.

Elements of $\mathbb{S}_{2}^{2}$ may be regarded as sequences $T_{0}, T_{1}, T_{2}, \ldots$ of periodic tilings of equilateral triangles of side length $2^{n}$, where the standard subdivision of triangles of side length $2^{n}$ into four of side length $2^{n-1}$ takes $T_{n}$ to $T_{n-1}$. The topology on $\mathbb{S}_{2}^{2}$ regards two such sequences as close if their $n$th terms are small translates of each other, for large $n$. The factor map from $\Omega_{\mathrm{a}}$ to $\mathbb{S}_{2}^{2}$ simply records the sequence of unlabeled level- $n$ supertilings (where we use the triangular tile versions of these tilings). Triangles $t$ of $T_{n}$ which appear at the centers of the next level always correspond to R1-triangles, with turns directing counterclockwise about $t$, so the map from $\Omega_{\mathrm{a}}$ to $\mathbb{S}_{2}^{2}$ is 1-to-1 everywhere except over points having an infinite-level vertex (that is, a point which is a vertex of every $T_{n}$ ). These points correspond to CHT tilings [21], where the map is 3 -to- 1 .

By Lemma 3.6, there are $2^{\ell}$ different ways of adding charges to a compatible R1-tiling to obtain a valid tiling in $\Omega$, where $\ell$ is the number of connected components of the R1edge graph. We determine this number $\ell$ in the following, and hence the multiplicity of the fibers of the factor maps to $\Omega_{\mathrm{a}}$ and the MEF:

Lemma 6.4. Set $T \in \Omega$ with $\ell$ connected components of the $\boldsymbol{R} 1$-edge graph (so $\ell=1,2$, or 3$)$. 

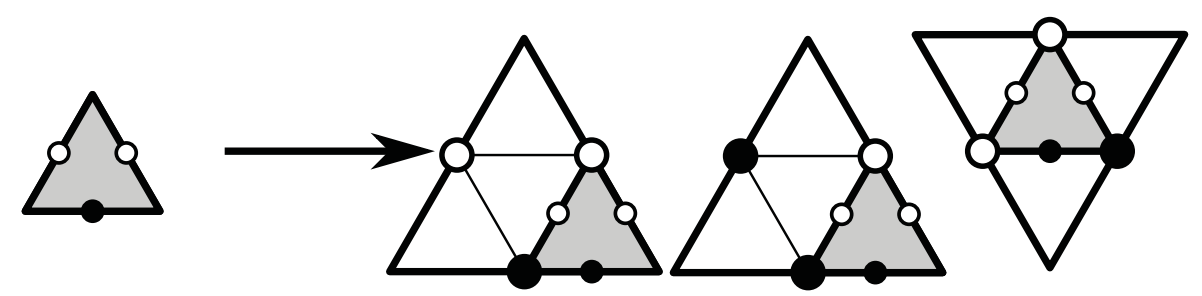

Figure 14. The $\alpha, \beta$, and $\gamma$ moves, in this respective order, used to create tilings with $\ell=2$ components of R1-edge graph. The white dots on the original tile indicate edges in the same R1-edge-graph component $X$, and the black dot indicates the other component $Y$. The three edges of the triangles at the next level are in the component indicated with larger dots.

(1) If $\ell=3$, then $T$ is a $\boldsymbol{C H T}$ tiling (possibly sheared along the bi-infinite $\boldsymbol{R} 1$-edge to make a fault line), a $P_{\infty}$ tiling, or an $n$-cycle. If $T \in \Omega_{\varphi}$, then $T$ is a $\boldsymbol{C H T}$ or $P_{\infty}$ tiling. There are eight assignments of charges to the $\boldsymbol{C H T}$ tiling and six for the $P_{\infty}$ tiling which give elements of $\Omega_{\varphi}$.

(2) If $\ell=2$ and $T$ has one bi-infinite $\boldsymbol{R} 1$-edge, then $T \notin \Omega_{\varphi}$. If $\ell=2$ and has no biinfinite $\boldsymbol{R} 1$-edge, then $T \in \Omega_{\varphi}$ and there is a sequence $\Delta_{0} \subset \Delta_{1} \subset \Delta_{2} \subset \cdots$ covering $\mathbb{R}^{2}$, where each $\Delta_{k}$ is a $k$-supertile which, for sufficiently large $k$, is included into $\Delta_{k+1}$ in one of the placements $\alpha, \beta$, or $\gamma$ depicted in Figure 14.

(3) If $\ell=1$, then either $\boldsymbol{R} \mathbf{1}(T)$ has an infinite fault line or $T \in \Omega_{\varphi}$. Almost every element of $\Omega$ has $\ell=1$.

Proof. It follows from Theorem 4.5 that $\ell=1,2$, or 3 . Suppose that $\ell=3$. If $T$ is a $P_{\infty}$ tiling or an $n$-cycle, then it is easily seen that $\ell=3$. Otherwise, by Proposition 5.4 , it has supertile decompositions. If $T$ is covered by a nested union of supertiles, then it must be admitted by $\varphi$. Otherwise, there must be a bi-infinite R1-edge with two infinite R1triangles each side, making it a CHT tiling, or a shear of it along the infinite R1-edge. If $T \in \Omega_{\varphi}$, then it follows that $T$ is a $P_{\infty}$ tiling or a CHT tiling. In the former case, there is a nested union $\Delta_{0} \subset \Delta_{1} \subset \Delta_{2} \subset \cdots$ where each $\Delta_{k}$ is a $k$-supertile (with inward-directed edges) appearing at the center of $\Delta_{k+1}$. There are six ways of labeling $\Delta_{0}$ with charges to obtain a prototile of $\mathcal{P}$, namely those which do not use the same charge on all edges, and this charge determines that of all of the other $\Delta_{k}$. For the CHT tiling, all eight valid assignments of charges give a tiling of $\Omega_{\varphi}$. This follows from a brief check that all eight possible charge assignments to two $\mathbf{R} 1$-triangles meeting opposite across an R1-edge can be generated by the substitution.

So now suppose that $\ell=2$. If $T$ has a bi-infinite R1-edge $E$, the R1-edge graph on one half-plane side of $E$ must be in the same component as $E$, whereas the other side must have an infinite R1-triangle. This can happen in $\Omega$ but not in $\Omega_{\varphi}$. Suppose then that $T$ does not have a bi-infinite $\mathbf{R} 1$-edge. Since $T$ is not an $n$-cycle (which has $\ell=3$ ), there must be the stated sequence $\Delta_{0} \subset \Delta_{1} \subset \Delta_{2} \subset \cdots$ of $k$-supertiles, by Proposition 5.4 ; indeed, if any such sequence covered only part of the plane, then there would be an infinite R1-line. All of the supertiles are substitutes of tiles from $\mathcal{P}$, or else the tiling 
would be a $P_{\infty}$ tiling. It follows that $T$ is admitted by $\varphi$. Since $\ell=2$, for sufficiently large $k$, two of the edges of $\Delta_{k}$ belong to the same component $X$ of the R1-edge graph, and the other edge belongs to the other component $Y$. This limits the placement of $\Delta_{k}$ into $\Delta_{k+1}$ to one of $\alpha, \beta$, or $\gamma$ in Figure 14 .

Finally, suppose that $\ell=1$. By Proposition 5.4, $T$ either is admitted by $\varphi$ or has an infinite R1-line, splitting into half-planes that are patches of certain tilings from $\Omega_{\varphi}$. If $\mathbf{R} \mathbf{1}(T)$ has a fault line, then $T \notin \Omega_{\varphi}$. If there is no fault line in the R1-decorations, then since $\ell=1$, the charges of one half-plane determine those on the other and $T$ itself must be admitted by $\varphi$. Finally, we claim that almost every element of $\Omega_{\varphi}$ (and hence $\Omega$, by Corollary 6.3 ) has $\ell=1$. Indeed, $k$-supertiles of all levels are positioned periodically, with two given sides being connected in the R1-edge graph at the next level whenever they appear in one of four possible locations in the $(k+1)$-supertile containing them. So generically, any two given edges are eventually connected in some sufficiently large supertile.

We can give a precise description of which tilings have $\ell=2$ connected components of R1-edge graph. A triangle (positioned with horizontal bottom edge) may have edges directed inward or outward, to which we associate a tuple $(l, r, b) \in\{i, o\}^{3}$, with the first, second, and third coordinates corresponding to the left, right, and bottom edges, respectively, and $o$ denoting 'outward' and $i$ denoting 'inward'. Because of the placement of triangles, we may only apply an $\alpha$ or $\beta$ move to an $(o, x, y)$ triangle (where $x, y \in\{0, i\}$ may be chosen arbitrarily), and a $\gamma$ move may only be applied to an $(i, i, i)$ triangle (in particular, we cannot have an $(i, x, y)$ triangle unless $x=y=i)$. Applying an $\alpha$ move to an $(o, x, y)$ triangle results in a $(z, x, y)$ triangle, whereas $\beta$ results in a $(y, z, x)$ triangle, both for $z$ arbitrary (where, for the latter, note that we rotate the resulting configuration, making $X$ the bottom edge, since in the new triangle the edges $Y$ are in the same component and take on the roles of the $X$-labeled edges from the step before). A $\gamma$ move can lead to any triangle type.

Thus, any path around the graph of Figure 15 builds an R1-tiling with $X$ and $Y$ in different path components. Moreover, so long as the path does not end in repeated applications of $\alpha$ (which would mean that $T$ is a CHT tiling, if $T \in \Omega_{\varphi}$ ) or of $\gamma$ (which would result in a $P_{\infty}$ tiling), it is easily seen that the sequence of supertiles covers the plane. Moreover, in this case, since two edges of a triangle are reconnected at either an $\alpha$ or a $\beta$ move, we see that the resulting tiling has precisely two connected components. Conversely, a valid tiling with two components (without bi-infinite R1-line) is covered by supertiles whose placements eventually follow an infinite path in the graph of Figure 15 and does not have an infinite tail of only $\alpha$ or only $\gamma$.

Having determined the multiplicities of fibers to $\Omega_{\mathrm{a}}$ (and the MEF), we now compare the situation to the Penrose $\left(1+\epsilon+\epsilon^{2}\right)$ - and Socolar-Taylor tilings, which, despite not being MLD (the Čech cohomologies of $\Omega_{\mathrm{ST}}$ and $\Omega_{\epsilon}$ differ [1]), somewhat amazingly map to the MEF with fibers of identical multiplicity $[1,11]$. We summarise with Figure 16.

In Figure 16, 'gen' is shorthand for 'generic' and 'sing' for 'singular'. The a-lines of a point in the solenoid (or associated tiling) are defined in [11]; they correspond to the locations of the (nonoffset) R1-edges, which are arranged periodically, and more sparsely 


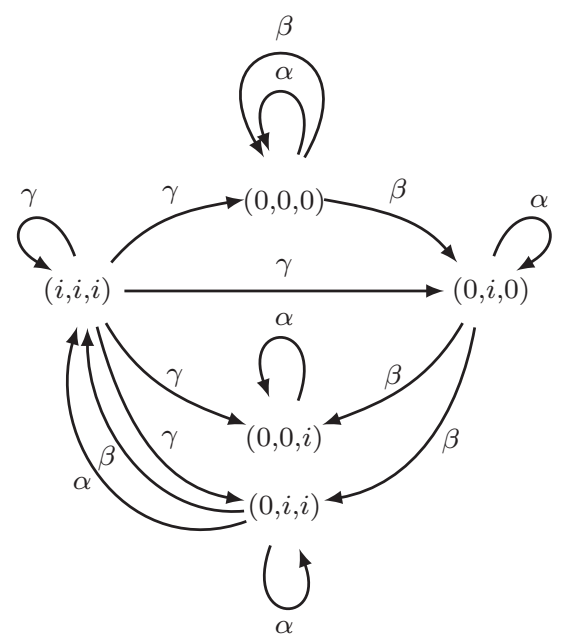

Figure 15. Graph of nodes of the different triangle types, connected by all possible $\alpha$, $\beta$, and $\gamma$ moves (see Figure 14). An infinite path in this graph which does not end in an infinite tail of $\alpha$ or infinite tail of $\gamma$ defines a tiling with $\ell=2$ components of R1-edge graph.

\begin{tabular}{|l||l|l|l|}
\hline \multicolumn{1}{|c||}{} & \multicolumn{2}{c|}{ Size of fibre of $* \rightarrow \Omega_{\mathrm{a}}$} & \\
\hline Type of $\mathbb{S}_{2}^{2}$ point & $\Omega_{\mathrm{ST}}$ and $\Omega_{\epsilon}$ & $\Omega_{\varphi}^{ \pm}$ & $\begin{array}{l}\text { Size of fibre of } \\
\Omega_{\mathrm{a}} \rightarrow \mathbb{S}_{2}^{2}\end{array}$ \\
\hline \hline $\begin{array}{l}\text { CHT (w and a- } \\
\text { sing) }\end{array}$ & 2 & 4 & 6 \\
\hline$P_{\infty}(\mathbf{i C W}-\mathbf{L})$ & 6 & 3 & 1 \\
\hline a-gen, w-gen & 1 & 1 or 2 & 1 \\
\hline a-sing, w-gen & 1 & 1 & 2 \\
\hline a-gen, w-sing & 2 & 1 or 2 & 1 \\
\hline
\end{tabular}

Figure 16. Comparing fiber multiplicities with the Penrose $\left(1+\epsilon+\epsilon^{2}\right)$ - and Socolar-Taylor tilings.

at increasing levels of the hierarchy. Tilings where every a-line has a finite level are called a-generic; otherwise they are a-singular (that is, a tiling is a-singular if it has an infinite R1-line). The w-lines are defined somewhat similarly. They form another triangular grid at each level, each line cutting through lines of reflective symmetry of the triangles of a-lines, and are related to the carry of information of flags (or colors) in the R2-rule of the Socolar-Taylor tilings. We refer the reader to [11] for more details on these notions. The values in the first column of Figure 16 are given in [11] and [1].

For the second column of values, the first two rows are the content of Lemma 6.4(1). For the third row it is not hard to see (analogous to the proof that almost all tilings have $\ell=1$ components of an R1-edge graph in the proof of Lemma 6.4(3)) that almost all tilings have no infinite a- or w-lines (these are also called generic in [11]). So almost every tiling is generic and has $\ell=1$, although we may also construct a- and $\mathrm{w}$ - generic tilings with $\ell=2$, which will happen for most infinite paths in the graph of Figure 15 (the path $\beta^{\infty}$ gives just one example). The fourth row (a-sing, w-gen) corresponds to tilings 
with an infinite R1-line which are not CHT, which have $\ell=1$ by Lemma 6.4. Finally, we may find tilings without infinite $\mathbf{R} 1$-edges but an infinite w-line with either $\ell=1$ or $\ell=2$. Following the path $\alpha \gamma^{6} \alpha \gamma^{6} \alpha \gamma^{6} \cdots$ of the graph in Figure 15 constructs an example with $\ell=2$. Examples with $\ell=1$ are simple to construct, by successively placing supertiles with common lines of reflection and not following a path in the graph of Figure 15.

Since $\Omega_{\varphi}^{ \pm}$factors almost everywhere 1-to- 1 to its MEF, it has pure point dynamical and diffraction spectrum (see, for example $[6,12]$ ), and a regular model set structure by $[3$, Theorem 6]. This factor map has a different multiplicity of fibers from $\Omega_{\mathrm{ST}}$ and $\Omega_{\epsilon}$ and so cannot be topologically conjugate to either of them. We summarize these main results together with results from $[1,11]$ :

Corollary 6.5. We have the following diagram of maps of $\mathbb{R}^{2}$-dynamical systems:

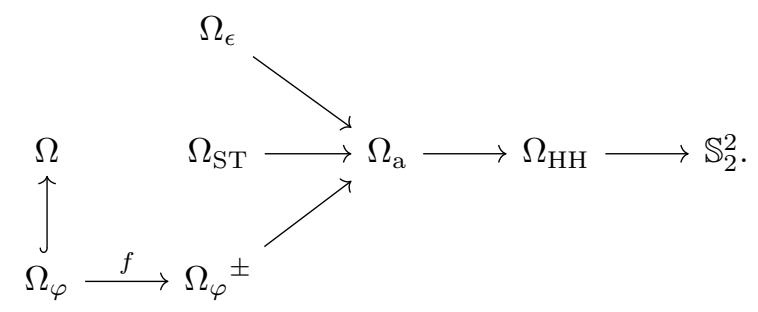

Each map is a factor map, except for the inclusion $\Omega_{\varphi} \hookrightarrow \Omega$, which has image of full measure. All factor maps are almost everywhere 1-to- 1 , except for the map $f$, which is a 2-to- 1 covering map. In particular, $\left(\Omega, \mathbb{R}^{2}\right)$ and $\left(\Omega_{\varphi}, \mathbb{R}^{2}\right)$ do not have pure point dynamical spectrum, but $\left(\Omega_{\varphi}^{ \pm}, \mathbb{R}^{2}\right)$ does. Moreover, none of these systems are topologically conjugate. The tiling spaces $\Omega_{\epsilon}, \Omega_{\mathrm{ST}}, \Omega_{\mathrm{a}}$, and $\Omega_{\varphi}^{ \pm}$each have the structure of regular model sets.

Acknowledgments. We thank the anonymous referee for their helpful comments. We are grateful to Franz Gähler for discussions which helped to uncover the substitution generating a full-measure subset of tilings in our hull. This research was partially supported by EPSRC grant EP/R013691/1.

Competing Interest. None.

\section{References}

[1] M. BaAke, F. Gähler and U. Grimm, Hexagonal inflation tilings and planar monotiles, Symmetry 4 (2012), 581-602.

[2] M. Bahke and U. Grimm, Aperiodic Order, Vol. 1, Encyclopedia of Mathematics and Its Applications, 149 (Cambridge University Press, Cambridge, 2013).

[3] M. BaAke, D. Lenz And R. V. Moody, Characterization of model sets by dynamical systems, Ergodic Theory Dynam. Systems 27 (2007), 341-382.

[4] M. Barge And J. Kellendonk, Proximality and pure point spectrum for tiling dynamical systems, Michigan Math. J. 62 (2013), 793-822.

[5] R. Berger, The undecidability of the domino problem, Mem. Amer. Math. Soc. 66 (1966). 
[6] S. Dworkin, Spectral theory and x-ray diffraction, J. Math. Phys. 34 (1993), 2965-2967.

[7] T. Fernique and N. Ollinger, Combinatorial substitutions and sofic tilings, in Proceedings of JAC 2010: Journées Automates Cellulaires, pp. 100-110 (Turku Centre for Computer Science, Turku, Finland, 2010).

[8] C. Goodman-Strauss, Matching rules and substitution tilings, Ann. of Math. (2) 147 (1998), 181-223.

[9] B. Grünbaum and G. C. Shephard, Tilings and Patterns (W. H. Freeman and Company, New York, 1987).

[10] P. Gummelt, Penrose tilings as coverings of congruent decagons, Geom. Dedicata 62 (1996), 1-17.

[11] J.-Y. Lee And R. V. Moody, Taylor-Socolar hexagonal tilings as model sets, Symmetry 5 (2013), 1-46.

[12] J.-Y. Lee, R. V. Moody and B. Solomyak, Pure point dynamical and diffraction spectra, Ann. Henri Poincaré 3 (2002), 1003-1018.

[13] M. Mampusti And M. F. Whittaker, An aperiodic monotile that forces nonperiodicity through dendrites Bull. Lond. Math. Soc. 52 (2020), 942-959.

[14] S. Mozes, Tilings, substitution systems and dynamical systems generated by them, $J$. Anal. Math. 53 (1989), 139-186.

[15] R. Penrose, Pentaplexity: A class of nonperiodic tilings of the plane, Math. Intelligencer $2(1979 / 80), 32-37$.

[16] R. Penrose, 'Twistor newsletters', 1996/97, http://people.maths.ox.ac.uk/lmason/Tn/41/TN41-08.pdf, http://people.maths.ox.ac.uk/lmason/Tn/42/TN42-09.pdf, http://people.maths.ox.ac.uk/lmason/Tn/43/TN43-11.pdf.

[17] R. Penrose, Remarks on tiling: Details of a $\left(1+\epsilon+\epsilon^{2}\right)$-aperiodic set, in The Mathematics of Long-Range Aperiodic Order (Waterloo, ON, 1995), NATO Advanced Science Institutes Series C: Mathematical and Physical Sciences, 489, pp. 467-497 (Kluwer Academic Publishers, Dordrecht, the Netherlands, 1997).

[18] R. M. Robinson, Undecidability and nonperiodicity for tilings of the plane, Invent. Math. 12 (1971), 177-209.

[19] L. Sadun, Topology of Tiling Spaces, University Lecture Series, 46 (American Mathematical Society, Providence, RI, 2008).

[20] D. Shechtman, I. Blech, D. Gratias and J. W. Cahn, Metallic phase with long-range orientational order and no translational symmetry, Phys. Rev. Lett. 53 (1984), 1951-1953.

[21] J. E. S. Socolar and J. M. TAYlor, An aperiodic hexagonal tile, J. Combin. Theory Ser. A 118 (2011), 2207-2231.

[22] J. E. S. Socolar And J. M. TAYlor, Forcing nonperiodicity with a single tile, Math. Intelligencer 34 (2012), 18-28.

[23] J. M. TAYLOR, Aperiodicity of a functional monotile, Preprint, (2010).

[24] H. Wang, Proving theorems by pattern recognition-II, Bell Syst. Tech. J. 40 (1961), 1-41. 